\title{
FC Sets and Twisters: The Basics of Orbifold Deconstruction
}

\author{
Peter Bantay (1) \\ Institute for Theoretical Physics, Eötvös Loránd University, Pázmány Péter s. 1/A, 1117 Budapest, \\ Hungary. E-mail: bantay@poe.elte.hu
}

Received: 10 December 2019 / Accepted: 21 July 2020

Published online: 15 September 2020 - (C) The Author(s) 2020

\begin{abstract}
We present a detailed account of the properties of twisters and their generalizations, FC sets, which are essential ingredients of the orbifold deconstruction procedure aimed at recognizing whether a given conformal model may be obtained as an orbifold of another one, and if so, to identify the twist group and the original model. The close analogy with the character theory of finite groups is discussed, and its origin explained.
\end{abstract}

\section{Introduction}

Orbifold deconstruction, i.e. the procedure aimed at recognizing whether a given 2D conformal model is an orbifold $[12,15]$ of another one, and if so, to identify (up to isomorphism) the relevant twist group and the original model, is an effective tool to better understand both the general properties of conformal models and the precise structure of their orbifolds. The basic ideas have been described in $[3,6]$, focusing on conceptual issues without going into the mathematical details. The purpose of the present paper is to fill this gap by giving a formal treatment of the concepts underlying the deconstruction procedure.

The starting point of orbifold deconstruction is the observation $[3,6]$ that every orbifold has a distinguished set of primaries, the so-called vacuum block, consisting of the descendants of the vacuum, and that this vacuum block has quite special properties: it is closed under the fusion product, and all its elements have integral conformal weight and quantum dimension. Such sets of primaries were termed 'twisters' because of their relation to twist groups and twisted boundary conditions. Twisters provide the input for the deconstruction procedure: to each different twister corresponds a different deconstruction, with possibly different twist groups and/or deconstructed models.

It turns out that most properties of twisters can be understood in the more general context of FC sets, which are those sets of primaries that are closed under the fusion product. As we shall see, these show deep analogies with character rings of finite groups, 
especially the so-called integral FC sets, all of whose elements have integral quantum dimension. In case of twisters, this analogy with character theory is of course far from being accidental, for it stems from their relation with the twist group of the corresponding orbifold, and it allows the generalization of several important group theoretic notions (like nilpotency, solubility, etc.) to general FC sets. In this respect, a most interesting question is: to what extent do classical results about groups generalize to selected classes of FC sets? We shall encounter several such conjectural generalizations on the way, e.g. of Lagrange's and Ito's celebrated theorems.

It should be pointed out that a special class of FC sets, the Abelian ones (cf. Definition 9) have been well-known for quite some time [23,30,31], their elements running under the name of simple currents, while the corresponding deconstructions are known as simple current extensions $[4,18]$. From this point of view it is fair to say that FC sets could be viewed as non-Abelian generalizations of simple current groups, and their theory bears the same relationship with that of simple currents as the representation theory of groups $[1,19,26]$ with that of Abelian ones.

In Sect. 2 we will review those standard results about the fusion ring of rational conformal models that form the basis of most of the subsequent arguments. Section 3 develops the basic theory of FC sets, introducing such fundamental notions as classes, blocks and their overlaps, and proving the modularity of the lattice of FC sets. Section 4 introduces the center of an FC set, and describes its basic properties, while the next section deals with central quotients and extensions. Section 6 is concerned with the arithmetic properties of FC sets, while Sect. 7 describes the structure of local FC sets and twisters, with their role in orbifold deconstruction expounded in Sect. 8. In our opinion, the highlights include the orthogonality relations Eqs. (3.6) and (3.7), the product rule (Theorem 1), Theorem 2 on the modularity of the lattice of FC sets, Theorem 6 on the structure of central quotients, and Theorem 7 on the integrality of quantum dimensions in local FC sets.

\section{Preliminaries}

Let's consider a rational unitary conformal model $[13,21,27]$. We will denote by $\mathrm{d}_{p}$ and $h_{p}$ the quantum dimension and conformal weight of a primary $p$, and by $\mathrm{N}(p)$ the associated fusion matrix, whose matrix elements are given by the fusion rules

$$
[\mathrm{N}(p)]_{q}^{r}=N_{p q}^{r}
$$

We will denote by 0 the vacuum primary, for which $\mathrm{d}_{0}=1, \mathrm{~h}_{0}=0$ and $\mathrm{N}(0)$ is the identity matrix. Note that, since

$$
\mathrm{N}(p) \mathrm{N}(q)=\sum_{r} N_{p q}^{r} \mathrm{~N}(r)
$$

the fusion matrices generate a commutative matrix algebra over $\mathbb{C}$, the Verlinde algebra $\mathcal{V}$, whose irreducible representations are all of dimension 1. According to Verlinde's famous formula [34]

$$
N_{p q}^{r}=\sum_{w} \frac{S_{p w} S_{q w} \overline{S_{r w}}}{S_{0 w}}
$$


relating the fusion rules to the modular $S$-matrix, to each primary $p$ corresponds an irrep $\boldsymbol{\rho}_{p}$ of $\mathcal{V}$ that assigns to the fusion matrix $\mathrm{N}(q)$ the complex number

$$
\boldsymbol{\rho}_{p}(q)=\frac{S_{q p}}{S_{0 p}}
$$

In view of Eq. (2.2) this gives

$$
\sum_{r} N_{p q}^{r} \boldsymbol{\rho}_{w}(r)=\boldsymbol{\rho}_{w}(p) \boldsymbol{\rho}_{w}(q)
$$

which is equivalent to

$$
\sum_{r} N_{p q}^{r} S_{r w}=\frac{S_{p w} S_{q w}}{S_{0 w}}
$$

The quantum dimensions of the primaries, i.e. the common Perron-Frobenius eigenvector [20] of the fusion matrices are given by $\mathrm{d}_{p}=\boldsymbol{\rho}_{0}(p)$, and one has the inequality

$$
\left|\boldsymbol{\rho}_{p}(q)\right| \leq \mathrm{d}_{q}
$$

Since the fusion matrices have integer matrix elements, it follows that their eigenvalues, i.e. the $\boldsymbol{\rho}_{p}(q)$ are all algebraic integers; in particular, all quantum dimensions $\mathrm{d}_{p}$ are algebraic integers, that may be shown to divide the algebraic integer $\sqrt{\sum_{p} \mathrm{~d}_{p}^{2}}=S_{00}^{-1}$.

Note that the matrix $S$ used above is actually an auxiliary quantity, since it can be determined fully from the fusion rules and conformal weights through the formula

$$
\frac{S_{p q}}{S_{00}}=\sum_{r} N_{p q}^{r} \mathrm{~d}_{r} \frac{\omega(p) \omega(q)}{\omega(r)}
$$

where

$$
\omega(p)=\exp \left(2 \pi i h_{p}\right)
$$

is the exponentiated conformal weight of the primary $p$.

Lemma 1. For primaries $p$ and $q$ such that $\left|\boldsymbol{\rho}_{p}(q)\right|=d_{q}, N_{p q}^{r}>0$ iff

$$
\frac{\omega(p) \omega(q)}{\omega(r)}=\frac{\boldsymbol{\rho}_{p}(q)}{d_{q}} .
$$

Proof. If Eq. (2.10) holds, then obviously $\left|\boldsymbol{\rho}_{p}(q)\right|=\mathrm{d}_{q}$, since the left-hand side has modulus 1. Conversely, according to Eq. (2.8)

$$
\sum_{r} N_{p q}^{r} \mathrm{~d}_{r} \frac{\omega(p) \omega(q)}{\omega(r)}=\frac{S_{p q}}{S_{00}}=\frac{S_{p q}}{S_{0 p}} \frac{S_{0 p}}{S_{00}}=\boldsymbol{\rho}_{p}(q) \mathrm{d}_{p}=\frac{\boldsymbol{\rho}_{p}(q)}{\mathrm{d}_{q}} \sum_{r} N_{p q}^{r} \mathrm{~d}_{r}
$$

hence for $\boldsymbol{\rho}_{p}(q) \neq 0$ one obtains

$$
0=\sum_{r} N_{p q}^{r} \mathrm{~d}_{r}\left(1-\frac{\omega(p) \omega(q) \mathrm{d}_{q}}{\omega(r) \boldsymbol{\rho}_{p}(q)}\right)
$$


or, after taking real parts

$$
\sum_{r} N_{p q}^{r} \mathrm{~d}_{r}\left(1-\operatorname{Re}\left(\frac{\omega(p) \omega(q) \mathrm{d}_{q}}{\omega(r) \boldsymbol{\rho}_{p}(q)}\right)\right)=0 .
$$

Since the real part of a complex number cannot exceed its modulus

$$
\operatorname{Re}\left(\frac{\omega(p) \omega(q) \mathrm{d}_{q}}{\omega(r) \boldsymbol{\rho}_{p}(q)}\right) \leq\left|\frac{\omega(p) \omega(q) \mathrm{d}_{q}}{\omega(r) \boldsymbol{\rho}_{p}(q)}\right|=1
$$

for $\left|\boldsymbol{\rho}_{p}(q)\right|=\mathrm{d}_{q}>0$, consequently all terms of the sum are non-negative, hence they should all vanish, proving the claim.

\section{FC Sets}

Definition 1. A set $\mathfrak{g}$ of primaries is fusion closed (an FC set for short) if it contains the vacuum primary 0 , and for all $\alpha, \beta \in \mathfrak{g}$

$$
\sum_{\gamma \in \mathfrak{g}} N_{\alpha \beta}^{\gamma} \mathrm{d}_{\gamma}=\mathrm{d}_{\alpha} \mathrm{a}_{\beta}
$$

Taking into account that quantum dimensions are positive real numbers, this is tantamount to the requirement that $N_{\alpha \beta}^{\gamma}>0$ and $\alpha, \beta \in \mathfrak{g}$ implies $\gamma \in \mathfrak{g}$. Notice that

$$
\mathrm{N}(\alpha) \mathrm{N}(\beta)=\sum_{\gamma \in \mathfrak{g}} N_{\alpha \beta}^{\gamma} \mathrm{N}(\gamma)
$$

for $\alpha, \beta \in \mathfrak{g}$ by Eq. (2.2), hence the fusion matrices $\mathrm{N}(\alpha)$ generate a subalgebra $\mathcal{V}_{\mathfrak{g}}$ of the Verlinde algebra. Since $\mathcal{V}$ is commutative, the irreps of the subalgebra $\mathcal{V}_{\mathfrak{g}}$ are among the different restrictions of the irreps $\boldsymbol{\rho}_{p}$ of $\mathcal{V}$.

Definition 2. Given an FC set $\mathfrak{g}$, a $\mathfrak{g}$-class $\mathrm{C}$ is the set of all those primaries $p$ whose associated irreps $\boldsymbol{\rho}_{p}$ coincide when restricted to the subalgebra $\mathcal{V}_{\mathfrak{g}}$; we shall denote by $\boldsymbol{\rho}_{\mathrm{C}}$ this common restriction, and by $\alpha(\mathrm{C})=\boldsymbol{\rho}_{\mathrm{C}}(\alpha)$ the value it assigns to an element $\alpha \in \mathfrak{g}$.

Clearly, the collection $\mathscr{C} \ell(\mathfrak{g})$ of $\mathfrak{g}$-classes is a partition of the set of all primaries, and one has $S_{\alpha p}=\alpha(\mathrm{C}) S_{0 p}$ for $\alpha \in \mathfrak{g}$ if the primary $p$ belongs to the class C $\in \mathscr{C} \ell(\mathfrak{g})$.

Lemma 2. The number of $\mathfrak{g}$-classes equals the cardinality of $\mathfrak{g}$ :

$$
|\mathscr{C} \ell(\mathfrak{g})|=|\mathfrak{g}| .
$$

Proof. As explained above, the irreps of the subalgebra $\mathcal{V}_{\mathfrak{g}}$ are among the different restrictions of the irreps of $\mathcal{V}$, i.e. the irreps $\boldsymbol{\rho}_{\mathrm{C}}$ corresponding to the classes. It follows that the number of classes equals the dimension (over $\mathbb{C}$ ) of $\mathcal{V}_{\mathfrak{g}}$, and the later equals the cardinality of $\mathfrak{g}$, because the fusion matrices are linearly independent.

Definition 3. The extent $\llbracket C \rrbracket$ of the class $C \in \mathscr{C} \ell(\mathfrak{g})$ is the algebraic number

$$
\llbracket \mathrm{C} \rrbracket=\frac{1}{\sum_{p \in \mathrm{C}} S_{0 p}^{2}}
$$




\section{Lemma 3.}

$$
\sum_{C \in \mathscr{C} \ell(\mathfrak{g})} \frac{1}{\llbracket C \rrbracket}=1
$$

Proof. This follows at once from the unitarity of the matrix $S$.

Orthogonality relations. For $\alpha, \beta \in \mathfrak{g}$

$$
\sum_{C \in \mathscr{C} \ell(\mathfrak{g})} \frac{\alpha(C) \overline{\beta(C)}}{\llbracket C \rrbracket}= \begin{cases}1 & \text { if } \alpha=\beta \\ 0 & \text { otherwise }\end{cases}
$$

Proof.

$$
\sum_{\mathrm{C} \in \mathscr{C} \ell(\mathfrak{g})} \frac{\alpha(\mathrm{C}) \overline{\beta(\mathrm{C})}}{\llbracket \mathrm{C} \rrbracket}=\sum_{\mathrm{C} \in \mathscr{C} \ell(\mathfrak{g})} \sum_{p \in \mathrm{C}} S_{0 p}^{2} \frac{S_{\alpha p}}{S_{0 p}} \frac{\overline{S_{\beta p}}}{S_{0 p}}=\sum_{p} S_{\alpha p} \overline{S_{p \beta}}=\delta_{\alpha, \beta}
$$

by the unitarity of the matrix $S$.

Second orthogonality. For any two classes $C_{1}, C_{2} \in \mathscr{C} \ell(\mathfrak{g})$

$$
\sum_{\alpha \in \mathfrak{g}} \alpha\left(C_{1}\right) \overline{\alpha\left(C_{2}\right)}= \begin{cases}\llbracket C_{1} \rrbracket & \text { if } C_{1}=C_{2} \\ 0 & \text { otherwise }\end{cases}
$$

Proof. Consider the square matrix

$$
X_{\alpha \mathrm{C}}=\frac{\alpha(\mathrm{C})}{\sqrt{\llbracket \mathrm{C} \rrbracket}}
$$

with rows indexed by the elements $\alpha \in \mathfrak{g}$ and columns by the classes $\mathrm{C} \in \mathscr{C} \ell(\mathfrak{g})$. But

$$
\sum_{\mathrm{C} \in \mathscr{C} \ell(\mathfrak{g})} X_{\alpha \mathrm{C}} \overline{X_{\beta \mathrm{C}}}=\delta_{\alpha, \beta}
$$

by Eq. (3.6), meaning that the matrix $X$ is unitary, which implies at once

$$
\sum_{\alpha \in \mathfrak{g}} \frac{\overline{\alpha\left(\mathrm{C}_{1}\right)} \alpha\left(\mathrm{C}_{2}\right)}{\sqrt{\llbracket \mathrm{C}_{1} \rrbracket \llbracket \mathrm{C}_{2} \rrbracket}}=\sum_{\alpha \in \mathfrak{g}} \overline{X_{\alpha \mathrm{C}_{1}}} X_{\alpha \mathrm{C}_{2}}= \begin{cases}1 & \text { if } \mathrm{C}_{1}=\mathrm{C}_{2} \\ 0 & \text { otherwise }\end{cases}
$$

Corollary 1. The cardinality of the class $C \in \mathscr{C l}(\mathfrak{g})$ is given by

$$
|C|=\frac{1}{\llbracket C \rrbracket} \sum_{\alpha \in \mathfrak{g}} \overline{\alpha(C)} \operatorname{Tr} N(\alpha)
$$

Proof. Since the trace of a matrix equals the sum of its eigenvalues,

$$
\operatorname{Tr} \mathrm{N}(\alpha)=\sum_{p} \boldsymbol{\rho}_{p}(\alpha)=\sum_{\mathrm{C} \in \mathscr{C} \ell(\mathfrak{g})} \sum_{p \in \mathrm{C}} \boldsymbol{\rho}_{p}(\alpha)=\sum_{\mathrm{C} \in \mathscr{C} \ell(\mathfrak{g})}|\mathrm{C}| \alpha(\mathrm{C})
$$

for $\alpha \in \mathfrak{g}$, and the result follows at once from (3.7). 
Lemma 4. The characteristic function of the class $C \in \mathscr{C} \ell(\mathfrak{g})$ reads

$$
\delta_{C}(p)=\frac{1}{\llbracket C \rrbracket} \sum_{\alpha \in \mathfrak{g}} \overline{\alpha(C)} \frac{S_{\alpha p}}{S_{0 p}}= \begin{cases}1 & \text { if } p \in C \\ 0 & \text { otherwise }\end{cases}
$$

Proof. The primary $p$ belongs to the class D $\in \mathscr{C} \ell(\mathfrak{g})$ iff $S_{\alpha p}=\alpha(\mathrm{D}) S_{0 p}$ for $\alpha \in \mathfrak{g}$, hence

$$
\frac{1}{\llbracket \mathrm{C} \rrbracket} \sum_{\alpha \in \mathfrak{g}} \overline{\alpha(\mathrm{C})} \frac{S_{\alpha p}}{S_{0 p}}=\frac{1}{\llbracket \mathrm{C} \rrbracket} \sum_{\alpha \in \mathfrak{g}} \overline{\alpha(\mathrm{C})} \alpha(\mathrm{D})= \begin{cases}1 & \text { if } \mathrm{D}=\mathrm{C} \\ 0 & \text { otherwise }\end{cases}
$$

by Eq. (3.7), proving the claim.

Lemma 5. For any class $C \in \mathscr{C} \ell(\mathfrak{g})$ one has

$$
\sum_{w \in C} S_{p w} \overline{S_{w q}}=\frac{1}{\llbracket C \rrbracket} \sum_{\alpha \in \mathfrak{g}} \overline{\alpha(C)} N_{\alpha p}^{q}
$$

Proof. It follows from Eqs. (2.3) and (3.9) that

$$
\begin{aligned}
\sum_{w \in \mathrm{C}} S_{p w} \overline{S_{w q}} & =\sum_{w} \delta_{\mathrm{C}}(w) S_{p w} \overline{S_{w q}}=\sum_{w} S_{p w} \overline{S_{q w}}\left\{\frac{1}{\llbracket \mathrm{C} \rrbracket} \sum_{\alpha \in \mathfrak{g}} \overline{\alpha(\mathrm{C})} \frac{S_{\alpha w}}{S_{0 w}}\right\} \\
& =\frac{1}{\llbracket \mathrm{C} \rrbracket} \sum_{\alpha \in \mathfrak{g}} \overline{\alpha(\mathrm{C})}\left\{\sum_{w} \frac{S_{p w} \overline{S_{q w}} S_{\alpha w}}{S_{0 w}}\right\}=\frac{1}{\llbracket \mathrm{C} \rrbracket} \sum_{\alpha \in \mathfrak{g}} \overline{\alpha(\mathrm{C})} N_{\alpha p}^{q} .
\end{aligned}
$$

The class containing the vacuum primary 0 is of special importance: we shall denote it by $\mathfrak{g}^{\perp}$, and call it the trivial class ${ }^{1}$. According to the previous definitions, $\alpha\left(\mathfrak{g}^{\perp}\right)=$ $\boldsymbol{\rho}_{0}(\alpha)=\mathrm{d}_{\alpha}$ for $\alpha \in \mathfrak{g}$.

\section{Lemma 6.}

$$
\llbracket \mathfrak{g}^{\perp} \rrbracket=\sum_{\alpha \in \mathfrak{g}} d_{\alpha}^{2}
$$

Proof. Since $\alpha\left(\mathfrak{g}^{\perp}\right)=\mathrm{d}_{\alpha}$ for $\alpha \in \mathfrak{g}$, one has

$$
\sum_{\alpha \in \mathfrak{g}} \mathrm{d}_{\alpha}^{2}=\sum_{\alpha \in \mathfrak{g}} \overline{\alpha\left(\mathfrak{g}^{\perp}\right)} \alpha\left(\mathfrak{g}^{\perp}\right)=\llbracket \mathfrak{g}^{\perp} \rrbracket
$$

according to Eq. (3.7).

The trivial class maximizes the product of size and extent.

Lemma 7. $|C| \llbracket C \rrbracket \leq \mid \mathfrak{g}^{\perp} \| \mathfrak{g}^{\perp} \rrbracket$ for every class $C \in \mathscr{C} \ell(\mathfrak{g})$.

\footnotetext{
1 As remarked by the referee, the trivial class corresponds to the braided commutant of the subcategory generated by the FC set.
} 
Proof. By Eq. (3.8)

$$
|\mathrm{C}| \llbracket \mathrm{C} \rrbracket=\sum_{\alpha \in \mathfrak{g}} \overline{\alpha(\mathrm{C})} \operatorname{Tr} \mathrm{N}(\alpha)
$$

Since the matrix $\mathrm{N}(\alpha)$ is non-negative and $|\alpha(\mathrm{C})| \leq \mathrm{d}_{\alpha}=\alpha\left(\mathfrak{g}^{\perp}\right)$

$$
\left|\mathrm{C} \| \mathrm{C} \rrbracket \leq \sum_{\alpha \in \mathfrak{g}}\right| \overline{\alpha(\mathrm{C})}\left|\operatorname{Tr} \mathrm{N}(\alpha) \leq \sum_{\alpha \in \mathfrak{g}} \alpha\left(\mathfrak{g}^{\perp}\right) \operatorname{Tr} \mathrm{N}(\alpha)=\right| \mathfrak{g}^{\perp} \llbracket \mathfrak{g}^{\perp} \rrbracket
$$

by the triangle inequality, taking into account that $\mid C \| C \rrbracket>0$.

Theorem 1. (Product rule) If $N_{p q}^{r}>0$ for some $p \in \mathfrak{g}^{\perp}$, then the primaries $q$ and $r$ belong to the same $\mathfrak{g}$-class.

Proof. Denoting by $\mathrm{C}$ the class of $q$, one has the obvious equality

$$
\sum_{r \notin \mathrm{C}} N_{p q}^{r} \mathrm{~d}_{r}=\sum_{r} N_{p q}^{r} \mathrm{~d}_{r}-\sum_{r \in \mathrm{C}} N_{p q}^{r} \mathrm{~d}_{r}=\mathrm{d}_{p} \mathrm{~d}_{q}-\sum_{r \in \mathrm{C}} N_{p q}^{r} \mathrm{~d}_{r} .
$$

On the other hand, by Eqs. (3.9) and (2.5)

$$
\begin{aligned}
& \sum_{r \in \mathrm{C}} N_{p q}^{r} \mathrm{~d}_{r}=\sum_{r} \delta_{\mathrm{C}}(r) N_{p q}^{r} \mathrm{~d}_{r}=\sum N_{p q}^{r} \frac{S_{0 r}}{S_{00}}\left\{\frac{1}{\llbracket \mathrm{C} \rrbracket} \sum_{\alpha \in \mathfrak{g}} \overline{\alpha(\mathrm{C})} \frac{S_{\alpha r}}{S_{0 r}}\right\} \\
& =\frac{1}{\llbracket \mathrm{C} \rrbracket} \sum_{\alpha \in \mathfrak{g}} \overline{\alpha(\mathrm{C})} \frac{S_{0 \alpha}}{S_{00}}\left\{\sum_{r} N_{p q}^{r} \frac{S_{\alpha r}}{S_{0 \alpha}}\right\}=\frac{1}{\llbracket \mathrm{C} \rrbracket} \sum_{\alpha \in \mathfrak{g}} \overline{\alpha(\mathrm{C})} \frac{S_{0 \alpha}}{S_{00}} \frac{S_{\alpha p}}{S_{0 \alpha}} \frac{S_{\alpha q}}{S_{0 \alpha}} .
\end{aligned}
$$

Since $S_{\alpha p}=\mathrm{d}_{\alpha} S_{0 p}=\mathrm{d}_{p} S_{0 \alpha}$ for $p \in \mathfrak{g}^{\perp}$ and $S_{\alpha q}=\alpha(\mathrm{C}) S_{0 q}$, this gives

$$
\sum_{r \in \mathrm{C}} N_{p q}^{r} \mathrm{~d}_{r}=\frac{1}{\llbracket \mathrm{C} \rrbracket} \sum_{\alpha \in \mathfrak{g}} \overline{\alpha(\mathrm{C})} \frac{S_{0 \alpha}}{S_{00}} \frac{S_{\alpha p}}{S_{0 \alpha}} \frac{S_{\alpha q}}{S_{0 \alpha}}=\mathrm{d}_{p} \frac{S_{0 q}}{S_{00}} \frac{1}{\llbracket \mathrm{C} \rrbracket} \sum_{\alpha \in \mathfrak{g}} \overline{\alpha(\mathrm{C})} \alpha(\mathrm{C})=\mathrm{d}_{p} \mathrm{~d}_{q}
$$

from which one concludes

$$
\sum_{r \notin \mathrm{C}} N_{p q}^{r} \mathrm{~d}_{r}=0
$$

Since all terms on the left-hand side are non-negative, it follows that they all have to vanish, i.e. $N_{p q}^{r}=0$ for $r \notin \mathrm{C}$.

Corollary 2. $\mathfrak{g}^{\perp}$ is an FC set.

Proof. If $p, q \in \mathfrak{g}^{\perp}$ and $N_{p q}^{r}>0$, then $r \in \mathfrak{g}^{\perp}$ by Theorem 1 .

Corollary 2 implies that all notions and results about an FC set $\mathfrak{g}$ go over verbatim to its dual FC set $\mathfrak{g}^{\perp}$. In particular, the set of primaries is partitioned into $\mathfrak{g}^{\perp}$-classes, which we shall call $\mathfrak{g}$-blocks (or simply blocks) to avoid confusion with $\mathfrak{g}$-classes.

Definition 4. For an FC set $\mathfrak{g} \in \mathscr{L}$, a $\mathfrak{g}$-block is a class of the dual FC set $\mathfrak{g}^{\perp}$. We will denote the collection $\mathscr{C} \ell\left(\mathfrak{g}^{\perp}\right)$ of $\mathfrak{g}$-blocks by $\mathcal{B} \ell(\mathfrak{g})$.

Lemma 8. The primaries $p$ and $q$ belong to the same $\mathfrak{g}$-block iff there exists $\alpha \in \mathfrak{g}$ such that $N_{\alpha p}^{q}>0$. 
Proof. Since $\mathfrak{g}^{\perp}$ is an FC set according to Corollary 2, the orthogonality relations apply to it. In particular, Eq. (3.7) takes the form

$$
\sum_{w \in \mathfrak{g}^{\perp}} \frac{S_{w p}}{S_{0 p}} \frac{\overline{S_{w q}}}{S_{0 q}}= \begin{cases}\llbracket \mathfrak{b} \rrbracket & \text { if } p \text { and } q \text { belong to the same block } \mathfrak{b} \in \mathcal{B} \ell(\mathfrak{g}) \\ 0 & \text { otherwise. }\end{cases}
$$

By Lemma 5, this means that $p$ and $q$ belong to the same block precisely when

$$
\sum_{w \in \mathfrak{g}^{\perp}} S_{w p} \overline{S_{w q}}=\frac{1}{\llbracket \mathfrak{g}^{\perp} \rrbracket} \sum_{\alpha \in \mathfrak{g}} \mathrm{d}_{\alpha} N_{\alpha p}^{q}>0 .
$$

Since the quantum dimensions $\mathrm{d}_{\alpha}$ are all positive, this is equivalent to $N_{\alpha p}^{q}>0$ for some $\alpha \in \mathfrak{g}$.

Corollary 3. The $\mathfrak{g}$-block containing the vacuum is $\mathfrak{g}$ itself: $\left(\mathfrak{g}^{\perp}\right)^{\perp}=\mathfrak{g}$.

Proof. Indeed, if $q$ belongs to the same block as the vacuum primary 0, then there exist $\alpha \in \mathfrak{g}$ such that $\delta_{q, \alpha}=N_{\alpha 0}^{q}>0$ by Lemma 8 .

\section{Lemma 9.}

$$
\llbracket \mathfrak{g} \rrbracket \llbracket \mathfrak{g}^{\perp} \rrbracket=\sum_{p} d_{p}^{2}
$$

Proof. By Eqs. (3.11) and (3.9)

$$
\begin{array}{r}
\llbracket \mathfrak{g} \rrbracket=\sum_{w \in \mathfrak{g}^{\perp}} \mathrm{d}_{w}^{2}=\sum_{w} \delta_{\mathfrak{g}^{\perp}}(w) \mathrm{d}_{w}^{2}=\sum_{w} \frac{1}{\llbracket \mathfrak{g}^{\perp} \rrbracket} \sum_{\alpha \in \mathfrak{g}} \mathrm{d}_{\alpha} \frac{S_{\alpha w}}{S_{0 w}} \mathrm{~d}_{w}^{2} \\
=\frac{1}{\llbracket \mathfrak{g}^{\perp} \rrbracket} \sum_{\alpha \in \mathfrak{g}} \mathrm{d}_{\alpha} \sum_{w} S_{\alpha w} \frac{S_{0 w}}{S_{00}^{2}}=\frac{S_{00}^{-2}}{\llbracket \mathfrak{g}^{\perp} \rrbracket} \sum_{\alpha \in \mathfrak{g}} \mathrm{d}_{\alpha} \delta_{\alpha 0}=\frac{1}{\llbracket \mathfrak{g}^{\perp} \rrbracket} \sum_{p} \mathrm{~d}_{p}^{2}
\end{array}
$$

proving the assertion.

The above results illustrate the inherent duality of FC sets: $\mathfrak{g}$ and $\mathfrak{g}^{\perp}$ determine each other, while their extents are, roughly speaking, reciprocal. This duality means that any result about FC sets holds simultaneously for $\mathfrak{g}$ and its dual $\mathfrak{g}^{\perp}$. In particular, any result proven about classes gives a corresponding result about blocks, and vice versa. This seemingly trivial observation turns out to be quite useful.

Lemma 10. If $\mathfrak{g}$ and $\mathfrak{h}$ are $F C$ sets such that $\mathfrak{h} \subseteq \mathfrak{g}$, then every $\mathfrak{h}$-class is a union of $\mathfrak{g}$-classes, in particular $\mathfrak{g}^{\perp} \subseteq \mathfrak{h}^{\perp}$, and every $\mathfrak{g}$-block is a union of $\mathfrak{h}$-blocks.

Proof. If the primaries $p$ and $q$ belong to the same $\mathfrak{g}$-class, i.e. if the restrictions to $\mathcal{V}_{\mathfrak{g}}$ of the irreps $\boldsymbol{\rho}_{p}$ and $\boldsymbol{\rho}_{q}$ coincide, then for $\mathfrak{h} \subseteq \mathfrak{g}$ their restrictions to $\mathcal{V}_{\mathfrak{h}}$ coincide as well, showing that every $\mathfrak{g}$-class is contained in a unique $\mathfrak{h}$-class, hence each $\mathfrak{h}$-class is the union of the $\mathfrak{g}$-classes that it contains. Since the $\mathfrak{g}$-class containing the vacuum primary 0 is $\mathfrak{g}^{\perp}$, while the $\mathfrak{h}$-class containing it is $\mathfrak{h}^{\perp}$, this gives $\mathfrak{g}^{\perp} \subseteq \mathfrak{h}^{\perp}$, and consequently every $\mathfrak{g}$-block (i.e. $\mathfrak{g}^{\perp}$-class) is a union of $\mathfrak{h}$-blocks $\left(\mathfrak{h}^{\perp}\right.$-classes) by the above argument.

It follows from Lemma 10 that every $\mathfrak{g}$-class is a union of $\mathfrak{g}$-blocks precisely when $\mathfrak{g} \subseteq \mathfrak{g}^{\perp}$. It turns out that such FC sets play a basic role in orbifold deconstruction $[3,6]$, hence they deserve a special name. 
Definition 5. An FC set $\mathfrak{g}$ is local if $\mathfrak{g} \subseteq \mathfrak{g}^{\perp}$.

We shall encounter local FC sets in the sequel on several occasions. A major feature of this notion explaining its special standing is that, as a consequence of Lemma 27 and a result of Deligne [11], the corresponding subalgebra $\mathcal{V}_{\mathfrak{g}}$ may be identified with the character ring of some finite group, hence results from character theory [24,26,32] go over to local FC sets. This observation allows the generalization of many group theoretic notions to arbitrary FC sets, and provides a host of non-trivial conjectural results that seem to hold in full generality. As an example, consider the following notion.

Definition 6. The central character of a class $C \in \mathscr{C} \ell(\mathfrak{g})$ is the complex valued function $\varpi_{\mathrm{C}}: \mathfrak{g} \rightarrow \mathbb{C}$ assigning to $\alpha \in \mathfrak{g}$ the value

$$
\varpi_{\mathrm{C}}(\alpha)=\frac{\llbracket \mathfrak{g}^{\perp} \rrbracket}{\llbracket \mathrm{C} \rrbracket} \frac{\alpha(\mathrm{C})}{\mathrm{d}_{\alpha}} .
$$

It is clear that the values of the central character are always algebraic numbers. For local FC sets this notion gives back the corresponding classical one from character theory, and by well known results $[24,26]$, the values taken in that case are actually algebraic integers. Surprisingly, this seems to be true for generic FC sets.

Conjecture 1. $\varpi_{C}(\alpha)$ is always an algebraic integer.

Remark 1. The truth of Conjecture 1 would imply that the ratios

$$
\frac{\llbracket \mathfrak{g}^{\perp} \rrbracket}{\llbracket \mathrm{C} \rrbracket}=\varpi_{\mathrm{C}}(0)
$$

are algebraic integers for every $\mathrm{C} \in \mathscr{C} \ell(\mathfrak{g})$, leading to the following (conjectural) analogue of Lagrange's theorem: if $\mathfrak{g}$ and $\mathfrak{h}$ are FC sets and $\mathfrak{h} \subseteq \mathfrak{g}$, then $\llbracket \mathfrak{h} \perp$ divides $\llbracket \mathfrak{g}^{\perp} \rrbracket$, i.e. their ratio is an algebraic integer.

The inclusion relation makes the collection $\mathscr{L}$ of FC sets partially ordered, with maximal element the set of all primaries, and minimal element the trivial FC set $\{0\}$ consisting of the vacuum primary solely. Because the intersection of two FC sets is obviously an FC set again, $\mathscr{L}$ is actually a finite lattice $[8,22]$.

Proposition 1. Given $F C$ sets $\mathfrak{g}$ and $\mathfrak{h}$, their join $\mathfrak{g} \vee \mathfrak{h}$ (the smallest $F C$ set that contains both of them) is given by

$$
\mathfrak{g} \vee \mathfrak{h}=\left(\mathfrak{g}^{\perp} \cap \mathfrak{h}^{\perp}\right)^{\perp}
$$

hence the map that sends each FC set $\mathfrak{g}$ to $\mathfrak{g}^{\perp}$ is an isomorphism between the lattice $\mathscr{L}$ and its dual.

Proof. Since $\mathfrak{g}, \mathfrak{h} \subseteq \mathfrak{g} \vee \mathfrak{h}$ by definition, Lemma 10 implies $(\mathfrak{g} \vee \mathfrak{h})^{\perp} \subseteq \mathfrak{g}^{\perp}, \mathfrak{h}^{\perp}$, hence $(\mathfrak{g} \vee \mathfrak{h})^{\perp} \subseteq \mathfrak{g}^{\perp} \cap \mathfrak{h}{ }^{\perp}$, that is $\left(\mathfrak{g}^{\perp} \cap \mathfrak{h}^{\perp}\right)^{\perp} \subseteq \mathfrak{g} \vee \mathfrak{h}$. On the other hand, $\mathfrak{g}^{\perp} \cap \mathfrak{h}^{\perp} \subseteq \mathfrak{g}^{\perp}, \mathfrak{h}^{\perp}$ hence $\mathfrak{g}, \mathfrak{h} \subseteq\left(\mathfrak{g}^{\perp} \cap \mathfrak{h}^{\perp}\right)^{\perp}$ again by Lemma 10 , or in other words $\mathfrak{g} \vee \mathfrak{h} \subseteq\left(\mathfrak{g}^{\perp} \cap \mathfrak{h}^{\perp}\right)^{\perp}$, proving the claim.

Theorem 2. The lattice $\mathscr{L}$ of FC sets is modular (even Arguesian), but usually not distributive. 
Proof. The map that assigns to each FC set $\mathfrak{g}$ the collection $\mathcal{B} \ell(\mathfrak{g})$ of its blocks is clearly an injective embedding of $\mathscr{L}$ into the partition lattice of the set of all primaries, hence one has to prove the assertion for the image of this homomorphism. To prove modularity (or the stronger Arguesian property) of the latter, all we have to show is that for any pair $\mathfrak{g}, \mathfrak{h} \in \mathscr{L}$, if $\mathfrak{b}_{1} \in \mathcal{B} \ell(\mathfrak{g})$ and $\mathfrak{b}_{2} \in \mathcal{B} \ell(\mathfrak{h})$ are blocks such that $\mathfrak{b}_{1} \cap \mathfrak{b}_{2} \neq \emptyset$, then there is a block $\mathfrak{b} \in \mathcal{B} \ell(\mathfrak{g} \vee \mathfrak{h})$ that contains both of them [25]. But Lemma 10 implies that in case $\mathfrak{g} \subseteq \mathfrak{g} \vee \mathfrak{h}$ there exists for each $\mathfrak{b}_{1} \in \mathcal{B} \ell(\mathfrak{g})$ a block $\mathfrak{B}_{1} \in \mathcal{B} \ell(\mathfrak{g} \vee \mathfrak{h})$ such that $\mathfrak{b}_{1} \subseteq \mathfrak{B}_{1}$, and a similar argument shows that for $\mathfrak{b}_{2} \in \mathcal{B} \ell(\mathfrak{h})$ there exists $\mathfrak{B}_{2} \in \mathcal{B} \ell(\mathfrak{g} \vee \mathfrak{h})$ such that $\mathfrak{b}_{2} \subseteq \mathfrak{B}_{2}$. Since $\mathfrak{b}_{1} \cap \mathfrak{b}_{2} \subseteq \mathfrak{B}_{1} \cap \mathfrak{B}_{2}$, and two blocks are either equal or disjoint, we get that $\mathfrak{B}_{1}=\mathfrak{B}_{2}$ if $\mathfrak{b}_{1} \cap \mathfrak{b}_{2} \neq \emptyset$, and obviously $\mathfrak{B}_{1}$ contains both $\mathfrak{b}_{1}$ and $\mathfrak{b}_{2}$. As to distributivity, it already fails for a holomorphic $\mathbb{Z}_{2}$-orbifold (e.g. the $S O(16)$ Wess-Zumino model at level 1).

Remark 2. A better understanding of the lattice theoretic properties of $\mathscr{L}$ would be highly desirable. We just mention that, while $\mathscr{L}$ is modular according to the above, it is usually neither atomic nor complemented. In particular, it is unclear whether $\mathscr{L}$ admits a coordinatization in the spirit of $[9,35]$. Another interesting question, inspired by the results of [29], is to find extra identities satisfied by $\mathscr{L}$.

Going back to general properties of FC sets, note that, according to Lemma 8, restricting the indices of the fusion matrices $\mathrm{N}(\alpha)$ to the primaries belonging to a given block $\mathfrak{b} \in \mathcal{B} \ell(\mathfrak{g})$ results in non-negative integer matrices $N_{\mathfrak{b}}(\alpha)$ that form a representation $\Delta_{\mathfrak{b}}$ of the subalgebra $\mathcal{V}_{\mathfrak{g}}$. As a consequence of Eq. (2.6), for any primary $w$ belonging to the class $\mathrm{C} \in \mathscr{C} \ell(\mathfrak{g})$ one has

$$
\sum_{q \in \mathfrak{b}} \mathrm{N}_{\mathfrak{b}}(\alpha)_{p}^{q} S_{w q}=\boldsymbol{\rho}_{w}(\alpha) S_{w p}=\boldsymbol{\rho}_{\mathrm{C}}(\alpha) S_{w p}
$$

for all $\alpha \in \mathfrak{g}$, reflecting the fact that $\Delta_{\mathfrak{b}}$ decomposes as a direct sum of the irreducible representations $\boldsymbol{\rho}_{\mathrm{C}}$.

Definition 7. The overlap $\langle\mathfrak{b}, C)$ of the block $\mathfrak{b} \in \mathcal{B} \ell(\mathfrak{g})$ and the class $C \in \mathscr{C} \ell(\mathfrak{g})$ is the multiplicity of the irrep $\boldsymbol{\rho}_{\mathrm{C}}$ in the irreducible decomposition of the integral representation $\Delta \mathfrak{b}$.

Lemma 11. The overlap $\langle\mathfrak{b}, C\rangle$ equals the rank of the minor $S_{\mathfrak{b} C}$ of the modular $S$-matrix obtained by restricting the row indices to $\mathfrak{b} \in \mathcal{B} \ell(\mathfrak{g})$ and the column indices to $C \in \mathscr{C l}(\mathfrak{g})$.

Proof. Since $\mathrm{N}_{\mathfrak{b}}(\alpha) S_{\mathfrak{b C}}=\boldsymbol{\rho}_{\mathrm{C}}(\alpha) S_{\mathfrak{b C}}$ by Eq. (3.15), the columns of $S_{\mathfrak{b C}}$ span the invariant subspace of $\Delta_{\mathfrak{b}}$ corresponding to the irrep $\boldsymbol{\rho}_{\mathrm{C}}$. As the latter appears with multiplicity $\langle\mathfrak{b}, \mathfrak{C}\rangle$ in $\Delta_{\mathfrak{b}}$, we get the assertion.

Corollary 4. $\langle\mathfrak{b}, C\rangle=1$ iff the minor $S_{\mathfrak{b} C}$ factorizes, i.e. there exist complex functions $\xi: C \rightarrow \mathbb{C}$ and $\eta: \mathfrak{b} \rightarrow \mathbb{C}$ such that $S_{p q}=\xi(p) \eta(q)$ for $p \in C$ and $q \in \mathfrak{b}$. In particular, $\langle\mathfrak{g}, C\rangle=1$ for every class $C \in \mathscr{C} \ell(\mathfrak{g})$, and $\left\langle\mathfrak{b}, \mathfrak{g}^{\perp}\right\rangle=1$ for all $\mathfrak{b} \in \mathcal{B} \ell(\mathfrak{g})$.

Proof. Since rank 1 matrices factorize, the first statement is a special case of Lemma 11, and because $S_{\alpha p}=\alpha(\mathrm{C}) S_{0 p}$ for $p \in \mathrm{C}$ and $\alpha \in \mathfrak{g}$, this implies at once $\langle\mathfrak{g}, \mathrm{C}\rangle=1$, while $\left\langle\mathfrak{b}, \mathfrak{g}^{\perp}\right\rangle=1$ follows from this by duality.

Lemma 12. For $\mathfrak{b} \in \mathcal{B} \ell(\mathfrak{g})$ one has

$$
\sum_{C \in \mathscr{C} \ell(\mathfrak{g})}\langle\mathfrak{b}, C\rangle=|\mathfrak{b}|
$$


and for $C \in \mathscr{C} \ell(\mathfrak{g})$

$$
\sum_{\mathfrak{b} \in \mathcal{B} \ell(\mathfrak{g})}\langle\mathfrak{b}, C\rangle=|C|
$$

Proof. To prove Eq. (3.16), observe that

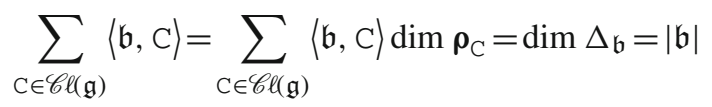

since $\operatorname{dim} \boldsymbol{\rho}_{\mathrm{C}}=1$. The second statement follows from this by duality.

\section{Lemma 13.}

$$
\langle\mathfrak{b}, C\rangle=\sum_{p \in \mathfrak{b}} \sum_{q \in C}\left|S_{p q}\right|^{2}
$$

Proof. Since the irrep $\boldsymbol{\rho}_{\mathrm{C}}$ of the subalgebra $\mathcal{V}_{\mathfrak{g}}$ appears with multiplicity $\langle\mathfrak{b}, C\rangle$ in the irreducible decomposition of $\Delta_{\mathfrak{b}}$, the matrix $\mathrm{N}_{\mathfrak{b}}(\alpha)$ has $\langle\mathfrak{b}, C\rangle$ eigenvalues equal to $\boldsymbol{\rho}_{\mathrm{C}}(\alpha)$ for $\alpha \in \mathfrak{g}$, hence

$$
\sum_{\mathrm{C} \in \mathscr{C} \ell(\mathfrak{g})}\langle\mathfrak{b}, \mathrm{C}\rangle \alpha(\mathrm{C})=\operatorname{Tr}\left(\mathrm{N}_{\mathfrak{b}}(\alpha)\right)=\sum_{p \in \mathfrak{b}} N_{\alpha p}^{p}
$$

i.e.

$$
\begin{array}{r}
\langle\mathfrak{b}, \mathrm{C}\rangle=\frac{1}{\llbracket \mathrm{C} \rrbracket} \sum_{\alpha \in \mathfrak{g}} \overline{\alpha(\mathrm{C})}\left\{\sum_{p \in \mathfrak{b}} N_{\alpha p}^{p}\right\}=\frac{1}{\llbracket \mathrm{C} \rrbracket} \sum_{\alpha \in \mathfrak{g}} \overline{\alpha(\mathrm{C})}\left\{\sum_{p \in \mathfrak{b}} \sum_{w} \frac{S_{\alpha w} S_{p w} \overline{S_{p w}}}{S_{0 w}}\right\} \\
=\sum_{p \in \mathfrak{b}} \sum_{w}\left|S_{p w}\right|^{2}\left\{\frac{1}{\llbracket \mathrm{C} \rrbracket} \sum_{\alpha \in \mathfrak{g}} \overline{\alpha(\mathrm{C})} \frac{S_{\alpha w}}{S_{0 w}}\right\}=\sum_{p \in \mathfrak{b}} \sum_{w}\left|S_{p w}\right|^{2} \delta_{\mathrm{C}}(w)=\sum_{p \in \mathfrak{b}} \sum_{q \in \mathrm{C}}\left|S_{p q}\right|^{2}
\end{array}
$$

using the orthogonality relation Eq. (3.7).

Corollary 5. $\langle\mathfrak{b}, C\rangle=0$ iff $S_{p q}=0$ for all $p \in \mathfrak{b}$ and $q \in C$.

Theorem 3. (Reciprocity relation) If the $F C$ sets $\mathfrak{g}, \mathfrak{h} \in \mathscr{L}$ satisfy $\mathfrak{g} \subseteq \mathfrak{h}$, then for every $\mathfrak{b} \in \mathcal{B} \ell(\mathfrak{h})$ and $C \in \mathscr{C} \ell(\mathfrak{g})$

$$
\sum_{\substack{\mathfrak{b}^{\prime} \in \mathcal{B} \ell(\mathfrak{g}) \\ \mathfrak{b}^{\prime} \subseteq \mathfrak{b}}}\left\langle\mathfrak{b}^{\prime}, C\right\rangle=\sum_{\substack{C^{\prime} \in \mathscr{C} \ell(\mathfrak{h}) \\ d^{\prime} \subseteq C}}\left\langle\mathfrak{b}, C^{\prime}\right\rangle .
$$

Proof. This follows from Lemma 10, since

$$
\begin{aligned}
& \sum_{\mathfrak{b}^{\prime} \in \mathcal{B} \ell(\mathfrak{g})}\left\langle\mathfrak{b}^{\prime}, \mathrm{C}\right\rangle=\sum_{\mathfrak{b}^{\prime} \in \mathcal{B} \ell(\mathfrak{g})} \sum_{p \in \mathfrak{b}^{\prime}}\left(\sum_{q \in \mathrm{C}}\left|S_{p q}\right|^{2}\right)=\sum_{p \in \mathfrak{b}} \sum_{q \in \mathrm{C}}\left|S_{p q}\right|^{2} \\
& \mathfrak{b}^{\prime} \subseteq \mathfrak{b} \\
& \mathfrak{b}^{\prime} \subseteq \mathfrak{b} \\
& =\sum_{C^{\prime} \in \mathscr{C} \ell(\mathfrak{h})} \sum_{q \in C^{\prime}}\left(\sum_{p \in \mathfrak{b}}\left|S_{p q}\right|^{2}\right)=\sum_{C^{\prime} \in \mathscr{C} \ell(\mathfrak{h})}\left\langle\mathfrak{b}, \mathrm{C}^{\prime}\right\rangle \\
& C^{\prime} \subseteq \mathrm{C} \\
& \mathrm{C}^{\prime} \subseteq \mathrm{C}
\end{aligned}
$$

as a consequence of Eq. (3.18). 
Corollary 6. If $\mathfrak{g} \subseteq \mathfrak{h}$, then the number of $\mathfrak{h}$-classes contained in $\mathfrak{g}^{\perp}$ equals the number of $\mathfrak{g}$-blocks contained in $\mathfrak{h}$.

Proof. Apply Corollary 4 and Eq. (3.19) with $C=\mathfrak{g}^{\perp}$ and $\mathfrak{b}=\mathfrak{h}$.

Proposition 2. For all $C \in \mathscr{C} \ell(\mathfrak{g})$ and $\mathfrak{b} \in \mathcal{B} \ell(\mathfrak{g})$ one has

$$
\langle\mathfrak{b}, C\rangle \leq \min \left(\frac{\llbracket \mathfrak{g}^{\perp} \rrbracket}{\llbracket C \rrbracket}, \frac{\llbracket \mathfrak{g} \rrbracket}{\llbracket \mathfrak{b} \rrbracket}\right) .
$$

Proof. Since

$$
\langle\mathfrak{b}, \mathrm{C}\rangle=\frac{1}{\llbracket C \rrbracket} \sum_{\alpha \in \mathfrak{g}} \overline{\alpha(\mathrm{C})} \operatorname{Tr} \mathrm{N}_{\mathfrak{b}}(\alpha)
$$

while $\operatorname{Tr} N_{\mathfrak{b}}(\alpha)$ is non-negative and $|\alpha(\mathrm{C})| \leq \mathrm{d}_{\alpha}$, one has

$$
\langle\mathfrak{b}, \mathrm{C}\rangle \leq \frac{1}{\llbracket \mathrm{C} \rrbracket} \sum_{\alpha \in \mathfrak{g}}\left|\overline{\alpha(\mathrm{C})} \operatorname{Tr} \mathrm{N}_{\mathfrak{b}}(\alpha)\right| \leq \frac{1}{\llbracket \mathrm{C} \rrbracket} \sum_{\alpha \in \mathfrak{g}} \mathrm{d}_{\alpha} \operatorname{Tr} \mathrm{N}_{\mathfrak{b}}(\alpha)=\frac{\llbracket \mathfrak{g}^{\perp} \rrbracket}{\llbracket \mathrm{C} \rrbracket}
$$

by the triangle inequality, taking into account Corollary 4 . Reversing the role of $\mathfrak{g}$ and $\mathfrak{g}^{\perp}$ completes the proof by duality.

Corollary 7. $\llbracket C \rrbracket \leq \llbracket \mathfrak{g}^{\perp} \rrbracket$ for $C \in \mathscr{C} \ell(\mathfrak{g})$, and $\llbracket \mathfrak{b} \rrbracket \leq \llbracket \mathfrak{g} \rrbracket$ for $\mathfrak{b} \in \mathcal{B} \ell(\mathfrak{g})$.

Proof. Indeed, $\llbracket C \rrbracket=\llbracket C \rrbracket|\mathfrak{g}, C\rangle \leq \llbracket \mathfrak{g}^{\perp} \rrbracket$ by Corollary 4 and Eq. (3.20). The second statement follows by duality.

The properties of those classes $C \in \mathscr{C l}(\mathfrak{g})$ that saturate the bound $\llbracket C \rrbracket \leq \llbracket \mathfrak{g}^{\perp} \rrbracket$ will be discussed in the next section.

Lemma 14. $|C| \leq \llbracket \mathfrak{g} \rrbracket$ for $C \in \mathscr{C} \ell(\mathfrak{g})$, and $|\mathfrak{b}| \leq \llbracket \mathfrak{g}^{\perp} \rrbracket$ for $\mathfrak{b} \in \mathcal{B} \ell(\mathfrak{g})$.

Proof. The first inequality follows from Eqs. (3.20) and (3.17), since

$$
|\mathrm{C}|=\sum_{\mathfrak{b} \in \mathcal{B} \ell(\mathfrak{g})}\langle\mathfrak{b}, \mathrm{C}\rangle \leq \sum_{\mathfrak{b} \in \mathcal{B} \ell(\mathfrak{g})} \frac{\llbracket \mathfrak{g} \rrbracket}{\llbracket \mathfrak{b} \rrbracket}=\llbracket \mathfrak{g} \rrbracket
$$

taking into account Eq. (3.5), and the second one follows by duality.

\section{The Center}

Definition 8. The center $\mathrm{z}(\mathfrak{g})$ of the FC set $\mathfrak{g} \in \mathscr{L}$ is the collection of those $\mathfrak{g}$-classes $z \in \mathscr{C l}(\mathfrak{g})$ for which $\llbracket z \rrbracket=\llbracket \mathfrak{g}^{\perp} \rrbracket$.

Clearly $\mathfrak{g}^{\perp} \in Z(\mathfrak{g})$, hence the center is never empty: we will call the elements of $\mathrm{Z}(\mathfrak{g})$ central classes. It follows from Proposition 2 that for a central class $z \in Z(\mathfrak{g})$ one has $\langle\mathfrak{b}, z\rangle \leq 1$ for any block $\mathfrak{b} \in \mathcal{B} \ell(\mathfrak{g})$, hence $|z| \leq\left|\mathfrak{g}^{\perp}\right|$ by Eq. (3.16).

Lemma 15. $z \in Z(\mathfrak{g})$ iff $|\alpha(z)|=d_{\alpha}$ for all $\alpha \in \mathfrak{g}$, i.e. the central character of $z$ has unit modulus. 
Proof. If $|\alpha(z)|=d_{\alpha}$ for all $\alpha \in \mathfrak{g}$, then

$$
\llbracket \mathrm{z} \rrbracket=\sum_{\alpha \in \mathfrak{g}}|\alpha(\mathrm{z})|^{2}=\sum_{\alpha \in \mathfrak{g}} \mathrm{d}_{\alpha}^{2}=\llbracket \mathfrak{g}^{\perp} \rrbracket
$$

by Eq. (3.7). Conversely, $\llbracket z \rrbracket=\llbracket \mathfrak{g}^{\perp} \rrbracket$ implies

$$
\sum_{\alpha \in \mathfrak{g}}\left(\mathrm{d}_{\alpha}^{2}-|\alpha(\mathrm{z})|^{2}\right)=0
$$

Since $|\alpha(z)|=\left|\rho_{z}(\alpha)\right| \leq d_{\alpha}$, all terms of the sum on the left-hand side are non-negative, hence they should all vanish.

Corollary 8. If $\mathfrak{g}, \mathfrak{h} \in \mathscr{L}$ are $F C$ sets such that $\mathfrak{h} \subseteq \mathfrak{g}$, then any class $C \in \mathscr{C l}(\mathfrak{h})$ containing a central class $z \in Z(\mathfrak{g})$ is itself central.

Proof. Since $\mathrm{z} \subseteq \mathrm{C}$, we have $\alpha(\mathrm{C})=\boldsymbol{\rho}_{\mathrm{C}}(\alpha)=\boldsymbol{\rho}_{\mathrm{z}}(\alpha)=\varpi_{\mathrm{z}}(\alpha) \mathrm{d}_{\alpha}$ for $\alpha \in \mathfrak{h}$, i.e. $|\alpha(\mathrm{z})|=$ $\mathrm{d}_{\alpha}$, proving the assertion according to Lemma 15 .

Definition 9. An FC set $\mathfrak{g} \in \mathscr{L}$ is Abelian if all its classes are central.

Proposition 3. An FC set $\mathfrak{g} \in \mathscr{L}$ is Abelian iff $d_{\alpha}=1$ for all $\alpha \in \mathfrak{g}$.

Proof. If all classes $\mathrm{C} \in \mathscr{C} \ell(\mathfrak{g})$ are central, then by Eq. (3.5)

$$
\llbracket \mathfrak{g}^{\perp} \rrbracket=\sum_{C \in \mathscr{C} \ell(\mathfrak{g})} \frac{\llbracket \mathfrak{g}^{\perp} \rrbracket}{\llbracket \mathrm{C} \rrbracket}=|\mathscr{C} \ell(\mathfrak{g})|=|\mathfrak{g}|
$$

hence by Eq. (3.11)

$$
0=\llbracket \mathfrak{g}^{\perp} \rrbracket-|\mathfrak{g}|=\sum_{\alpha \in \mathfrak{g}}\left(d_{\alpha}^{2}-1\right) .
$$

Since $\mathrm{d}_{\alpha} \geq 1$, we get the only if part. On the other hand, if $\mathrm{d}_{\alpha}=1$ for all $\alpha \in \mathfrak{g}$, then $\llbracket \mathfrak{g}^{\perp} \rrbracket=|\mathfrak{g}|$, hence by Eqs. (3.5) and (3.3)

$$
\sum_{C \in \mathscr{C} \ell(\mathfrak{g})}\left(\frac{1}{\llbracket C \rrbracket}-\frac{1}{\llbracket \mathfrak{g}^{\perp} \rrbracket}\right)=0 .
$$

Since all terms of the sum are non-negative by Corollary 7, they must all vanish.

Remark 3. In the language of 2D CFT, Abelian FC sets are groups of simple currents.

Lemma 16. If $\alpha, \beta, \gamma \in \mathfrak{g}$ are such that $N_{\alpha \beta}^{\gamma}>0$ and $z \in Z(\mathfrak{g})$ is a central class, then $\varpi_{z}(\gamma)=\varpi_{z}(\alpha) \varpi_{z}(\beta)$.

Proof. By definition of the central character

$$
\varpi_{\mathrm{z}}(\alpha) \varpi_{\mathrm{z}}(\beta) \mathrm{d}_{\alpha} \mathrm{d}_{\beta}=\alpha(\mathrm{z}) \beta(\mathrm{z})=\sum_{\gamma \in \mathfrak{g}} N_{\alpha \beta}^{\gamma} \gamma(\mathrm{z})=\sum_{\gamma \in \mathfrak{g}} N_{\alpha \beta}^{\gamma} \varpi_{\mathrm{z}}(\gamma) \mathrm{d}_{\gamma}
$$

which is equivalent to

$$
\sum_{\gamma \in \mathfrak{g}} N_{\alpha \beta}^{\gamma} \mathrm{d}_{\gamma}\left(1-\frac{\varpi_{z}(\gamma)}{\varpi_{z}(\alpha) \varpi_{z}(\beta)}\right)=0 .
$$

Since the real part of a complex number cannot exceed its modulus, all terms of the sum should equal 0. 
Lemma 17. If $p$ and $q$ are primaries such that $N_{\alpha p}^{q}>0$ for some $\alpha \in \mathfrak{g}$, then $p$ belongs to the central class $z \in Z(\mathfrak{g})$ iff

$$
\frac{\omega(\alpha) \omega(p)}{\omega(q)}=\varpi_{z}(\alpha) .
$$

Proof. This follows at once from Lemmas 15 and 1.

\section{Corollary 9.}

$$
\mathfrak{g}^{\perp}=\left\{p \mid \omega(q)=\omega(\alpha) \omega(p) \text { if } N_{\alpha p}^{q}>0 \text { for } \alpha \in \mathfrak{g}\right\}
$$

Proposition 4. If $C$ is a $\mathfrak{g}$-class and $\mathrm{z} \in Z(\mathfrak{g})$, then there exist unique $\mathfrak{g}$-classes $z^{ \pm 1} C \in$ $\mathscr{C l}(\mathfrak{g})$ such that

$$
\alpha\left(z^{ \pm 1} C\right)=\varpi_{z}(\alpha)^{ \pm 1} \alpha(C)
$$

for all $\alpha \in \mathfrak{g}$; in particular, $\mathfrak{g}^{\perp} C=$ C. Moreover,

$$
\llbracket z^{ \pm 1} C \rrbracket=\llbracket C \rrbracket
$$

and

$$
\varpi_{z C}(\alpha)=\varpi_{z}(\alpha) \varpi_{C}(\alpha) \text {. }
$$

Proof. According to Lemma 16, one has

$$
\begin{array}{r}
\sum_{\gamma \in \mathfrak{g}} N_{\alpha \beta}^{\gamma} \varpi_{\mathrm{z}}(\gamma)^{ \pm 1} \gamma(\mathrm{C})=\left\{\varpi_{\mathrm{z}}(\alpha) \varpi_{\mathrm{z}}(\beta)\right\}^{ \pm 1} \sum_{\gamma \in \mathfrak{g}} N_{\alpha \beta}^{\gamma} \gamma(\mathrm{C}) \\
=\left\{\varpi_{\mathrm{z}}(\alpha)^{ \pm 1} \alpha(\mathrm{C})\right\}\left\{\varpi_{\mathrm{z}}(\beta)^{ \pm 1} \beta(\mathrm{C})\right\}
\end{array}
$$

which means that the product $\varpi_{\mathrm{z}}^{ \pm 1} \boldsymbol{\rho}_{\mathrm{C}}$ is itself an irrep of the algebra $\mathcal{V}_{\mathfrak{g}}$, hence it is equal to the irrep corresponding to some well defined $\mathfrak{g}$-class, namely $\mathrm{z}^{ \pm 1} \mathrm{C}$. That $\mathfrak{g}^{\perp} \mathrm{C}=\mathrm{C}$ follows from $\varpi_{\mathfrak{g}^{\perp}}(\alpha)=1$ for $\alpha \in \mathfrak{g}$. Finally, Eq. (3.7) gives

$$
\llbracket \mathrm{z}^{ \pm 1} \mathrm{C} \rrbracket=\sum_{\alpha \in \mathfrak{g}}\left|\alpha\left(\mathrm{z}^{ \pm 1} \mathrm{C}\right)\right|^{2}=\sum_{\alpha \in \mathfrak{g}}|\alpha(\mathrm{C})|^{2}=\llbracket \mathrm{C} \rrbracket
$$

proving Eq. (4.3), leading to Eq. (4.4) when combined with Eq. (4.2).

Note the following generalization of the product rule Theorem 1.

Theorem 4. If $p$ belongs to the class $C \in \mathscr{C} \ell(\mathfrak{g})$ and $q$ belongs to the central class $z \in Z(\mathfrak{g})$, then $N_{p q}^{r}>0$ implies $r \in z C$.

Proof. According to Eqs. (3.9), (4.4) and (3.7)

$$
\begin{array}{r}
\sum_{r \in \mathrm{ZC}} N_{p q}^{r} \mathrm{~d}_{r}=\sum_{r} \delta_{\mathrm{zC}}(r) N_{p q}^{r} \mathrm{~d}_{r}=\frac{1}{\llbracket \mathrm{zC} \rrbracket} \sum_{\alpha \in \mathfrak{g}} \overline{\alpha(\mathrm{zC})} \sum_{r} \frac{S_{\alpha r}}{S_{0 r}} N_{p q}^{r} \mathrm{~d}_{r}= \\
\frac{1}{\llbracket \mathrm{C} \rrbracket} \sum_{\alpha \in \mathfrak{g}} \overline{\alpha(\mathrm{C})} \varpi_{\mathrm{z}}(\alpha)^{-1} \sum_{r} N_{p q}^{r} \frac{S_{\alpha r}}{S_{00}}=\frac{1}{\llbracket \mathrm{C} \rrbracket} \sum_{\alpha \in \mathfrak{g}} \overline{\alpha(\mathrm{C})} \varpi_{\mathrm{z}}(\alpha) \\
=\frac{1}{\llbracket \mathrm{C} \rrbracket} \sum_{\alpha \in \mathfrak{g}} \overline{S_{\alpha p}} \frac{S_{\alpha q}}{S_{\alpha 0}} \\
S_{00} \varpi_{\mathrm{z}}(\alpha)^{-1} \frac{\alpha(\mathrm{C}) S_{0 p}}{S_{\alpha 0}} \frac{\alpha(\mathrm{z}) S_{0 q}}{S_{00}}=\frac{\mathrm{d}_{p} \mathrm{~d}_{q}}{\llbracket \mathrm{C} \rrbracket} \sum_{\alpha \in \mathfrak{g}} \overline{\alpha(\mathrm{C})} \alpha(\mathrm{C})=\mathrm{d}_{p} \mathrm{~d}_{q}
\end{array}
$$


hence

$$
\sum_{r \notin z \mathrm{C}} N_{p q}^{r} \mathrm{~d}_{r}=0 .
$$

Since all terms on the left-hand side are non-negative, $N_{p q}^{r}=0$ for $r \notin \mathrm{zC}$.

Proposition 5. If $z_{1}, z_{2} \in Z(\mathfrak{g})$ are central classes, then $z_{1} z_{2}=z_{2} z_{1}$ is also central, and $\left(z_{1} z_{2}\right) C=z_{1}\left(z_{2} C\right)$ for all $C \in \mathscr{C} \ell(\mathfrak{g})$.

Proof. If $z_{1}, z_{2} \in \mathrm{Z}(\mathfrak{g})$, then $\llbracket z_{1} z_{2} \rrbracket=\llbracket z_{2} \rrbracket=\llbracket \mathfrak{g}^{\perp} \rrbracket$ by Eq. (4.3), proving that $z_{1} z_{2} \in$ $\mathrm{z}(\mathfrak{g})$. By Eq. (4.4), $\varpi_{z_{1} z_{2}}(\alpha)=\varpi_{z_{1}}(\alpha) \varpi_{z_{2}}(\alpha)=\varpi_{z_{2}}(\alpha) \varpi_{z_{1}}(\alpha)=\varpi_{z_{2} z_{1}}(\alpha)$ for $\alpha \in \mathfrak{g}$, and because central characters of different classes differ from each other, this shows that $z_{1} z_{2}=z_{2} z_{1}$. Finally, again by Eq. (4.4)

$$
\varpi_{\left(z_{1} z_{2}\right) C}(\alpha)=\varpi_{z_{1} z_{2}}(\alpha) \varpi_{C}(\alpha)=\varpi_{z_{1}}(\alpha) \varpi_{z_{2}}(\alpha) \varpi_{C}(\alpha)=\varpi_{z_{1}}(\alpha) \varpi_{z_{2} C}(\alpha)
$$

for $\mathrm{C} \in \mathscr{C} \ell(\mathfrak{g})$, hence $\left(\mathrm{z}_{1} \mathrm{z}_{2}\right) \mathrm{C}=\mathrm{z}_{1}\left(\mathrm{z}_{2} \mathrm{C}\right)$, finishing the proof.

Theorem 5. The center $Z(\mathfrak{g})$ of an $F C$ set $\mathfrak{g} \in \mathscr{L}$ is an Abelian group that permutes the $\mathfrak{g}$-classes.

Proof. Defining $z_{1} z_{2}$ as the product of the central classes $z_{1}, z_{2} \in z(\mathfrak{g})$, Proposition 5 implies that it is commutative and associative. Since $\mathfrak{g}^{\perp} z=z$ for every $z \in z(\mathfrak{g}), \mathfrak{g}^{\perp}$ is the identity element of this product, and the class $z^{-1} \mathfrak{g}^{\perp} \in Z(\mathfrak{g})$ is clearly the inverse of $z$, since $z\left(z^{-1} \mathfrak{g}^{\perp}\right)=\mathfrak{g}^{\perp}$, proving that $\mathrm{z}(\mathfrak{g})$ is indeed an Abelian group. Finally, again by Proposition 5 the maps $\mathrm{C} \mapsto \mathrm{zC}$ define a permutation action of $\mathrm{Z}(\mathfrak{g})$ on the set $\mathscr{C} \ell(\mathfrak{g})$ of $\mathfrak{g}$-classes.

\section{Central Quotients and Extensions}

Proposition 6. For an $F C$ set $\mathfrak{g}$ and a subgroup $Z<Z(\mathfrak{g})$ of its center,

$$
\mathfrak{g} / Z=\left\{\alpha \in \mathfrak{g} \mid \alpha(z)=d_{\alpha} \text { for all } z \in Z\right\}
$$

is again an $F C$ set, the central quotient of $\mathfrak{g}$ by $Z$, with dual

$$
(\mathfrak{g} / Z)^{\perp}=\bigcup_{z \in Z} z
$$

If $\mathfrak{h} \in \mathscr{L}$ is such that $\mathfrak{g} / Z \subseteq \mathfrak{h} \subseteq \mathfrak{g}$, then $\mathfrak{h}=\mathfrak{g} / H$ for some subgroup $H<Z$.

Proof. To simplify notation, let $\mathfrak{g}_{1}$ denote $\mathfrak{g} / Z$, and $\cup Z$ the union of the classes in $Z$. It follows from Lemma 16 that $N_{\alpha \beta}^{\gamma}>0$ for $\alpha, \beta \in \mathfrak{g}_{1}=\left\{\alpha \in \mathfrak{g} \mid \varpi_{\mathrm{Z}}(\alpha)=1\right.$ for all $\left.\mathrm{z} \in Z\right\}$ implies $\gamma \in \mathfrak{g}_{1}$, hence $\mathfrak{g}_{1} \in \mathscr{L}$. Clearly, $\cup Z \subseteq \mathfrak{g}_{1}^{\perp}$ since $\alpha(\mathrm{z})=\mathrm{d}_{\alpha}$ for $\mathrm{z} \in Z$ and $\alpha \in \mathfrak{g}_{1}$, while

$$
\llbracket \mathfrak{g}_{\mathbf{1}}^{\perp} \rrbracket=\sum_{\alpha \in \mathfrak{g}_{1}} \mathrm{~d}_{\alpha}^{2}=\sum_{\alpha \in \mathfrak{g}} \frac{1}{|Z|} \sum_{\mathrm{z} \in Z} \varpi_{\mathrm{z}}(\alpha) \mathrm{d}_{\alpha}^{2}=\frac{1}{|Z|} \sum_{\mathrm{z} \in Z} \sum_{\alpha \in \mathfrak{g}} \alpha(\mathrm{z}) \mathrm{d}_{\alpha}=\frac{\llbracket \mathfrak{g}^{\perp} \rrbracket}{|Z|}
$$

by Eqs. (3.11) and (3.7). Since $\llbracket z \rrbracket=\llbracket \mathfrak{g}^{\perp} \rrbracket$ for all $z \in Z(\mathfrak{g})$, one gets

$$
\sum_{p \in \mathfrak{g}_{\perp}^{\perp} \backslash \cup Z} S_{0 p}^{2}=\sum_{p \in \mathfrak{g}_{\perp}^{\perp}} S_{0 p}^{2}-\sum_{p \in \cup Z} S_{0 p}^{2}=\frac{1}{\llbracket \mathfrak{g}_{\mathbf{1}}^{\perp} \rrbracket}-\sum_{\mathrm{z} \in Z} \frac{1}{\llbracket z \rrbracket}=\frac{|Z|}{\llbracket \mathfrak{g}^{\perp} \rrbracket}-|Z| \frac{1}{\llbracket \mathfrak{g}^{\perp} \rrbracket}=0
$$


which implies that $\mathfrak{g}_{\mathbf{1}}^{\perp} \backslash \cup Z$ is void, since $S_{0 p}^{2}>0$ for all $p$.

Finally, if $\mathfrak{g}_{\mathbf{1}} \subseteq \mathfrak{h} \subseteq \mathfrak{g}$ then $\mathfrak{g}^{\perp} \subseteq \mathfrak{h}^{\perp} \subseteq \mathfrak{g}_{1}^{\perp}$ by Lemma 10 , hence $\mathfrak{h}^{\perp}$ is a union of $\mathfrak{g}$-classes contained in $\mathfrak{g}_{\mathfrak{1}}^{\perp}=\cup Z$; consequently, $\mathfrak{h}^{\perp}=\cup H$ for some subset $H \subseteq Z$, and because $\mathfrak{h}^{\perp}$ is an FC set, $H$ is actually a subgroup of $Z$ such that $(\mathfrak{g} / H)^{\perp}=\cup H=\mathfrak{h}^{\perp}$ by Eq. (5.2), i.e. $\mathfrak{h}=\mathfrak{g} / H$.

It follows from the above result that there is an order reversing one-to-one correspondence between central quotients of $\mathfrak{g} \in \mathscr{L}$ and subgroups of its center $\mathrm{Z}(\mathfrak{g})$. The usefulness of central quotients rests on the following result.

Theorem 6. For a subgroup $Z<Z(\mathfrak{g})$ of the center of $\mathfrak{g} \in \mathscr{L}$, let $\hat{Z}=\operatorname{Hom}\left(Z, \mathbb{C}^{\times}\right)$denote its character group (Pontryagin dual), and let $\mathfrak{g}_{\xi}=\left\{\alpha \in \mathfrak{g} \mid \varpi_{Z}(\alpha)=\xi(z)\right.$ for $\left.z \in Z\right\}$ for $\xi \in \hat{Z}$. Then

(1) each $\mathfrak{g}_{\xi}$ is a block of $\mathfrak{g} / Z$, of cardinality

$$
\left|\mathfrak{g}_{\xi}\right|=\frac{1}{|Z|} \sum_{z \in Z} \overline{\xi(z)}|\operatorname{Fix}(z)|
$$

where $\operatorname{Fix}(z)=\{C \in \mathscr{C l}(\mathfrak{g}) \mid z C=C\}$ denotes the set of fixed points of the central class $z \in Z(\mathfrak{g})$ in its action on $\mathscr{C} \ell(\mathfrak{g})$;

(2) $\llbracket \mathfrak{g}_{\xi} \rrbracket=\mid Z \llbracket \llbracket \mathfrak{g} \rrbracket$ for $\xi \in \hat{Z}$, i.e. each $\mathfrak{g}_{\xi}$ belongs to the center of $(\mathfrak{g} / Z)^{\perp}$, and $\mathfrak{g}_{\xi} \mathfrak{g}_{\eta}=\mathfrak{g}_{\xi \eta}$ for $\xi, \eta \in \hat{Z}$, hence $Z^{\perp}=\left\{\mathfrak{g}_{\xi} \mid \xi \in \hat{Z}\right\}$ is a subgroup of the center of $(\mathfrak{g} / Z)^{\perp}$ isomorphic to $Z$, and $(\mathfrak{g} / Z)^{\perp} / Z^{\perp}=\mathfrak{g}^{\perp}$

(3) each $\mathfrak{g} / Z$-class is of the form $Z C=\bigcup_{z \in Z} z C$ for some class $C \in \mathscr{C} \ell(\mathfrak{g})$, with $\llbracket \rrbracket=$ $\left[Z: Z_{C}\right] \llbracket Z C \rrbracket$ and

$$
\left\langle\mathfrak{g}_{\xi}, Z C\right\rangle= \begin{cases}1 & \text { if } \xi(z)=1 \text { for all } z \in Z_{C} \\ 0 & \text { otherwise }\end{cases}
$$

where $Z_{C}=\{z \in Z \mid z C=C\}$ denotes the stabilizer of $C$.

Proof. Lemma 16 implies that if $N_{\alpha \beta}^{\gamma}>0$ for $\alpha \in \mathfrak{g}_{\xi}$ and $\beta \in \mathfrak{g}_{\eta}$, then $\gamma \in \mathfrak{g}_{\xi \eta}$. In particular, if $\mathbf{I}$ denotes the principal character of $Z$ (the identity of $\hat{Z}$ ), then $\mathfrak{g}_{\mathbf{1}}$ is an FC set, and it follows from Lemma 8 that $\mathfrak{g}_{\xi} \in \mathcal{B} \ell\left(\mathfrak{g}_{1}\right)$ for each $\xi \in \hat{Z}$. Since clearly $\mathfrak{g}_{\mathbf{1}}=\mathfrak{g} / Z$, we get the first assertion. Next, notice that for $z \in Z(\mathfrak{g})$ one has by Eq. (3.7)

$$
\begin{aligned}
& |\mathrm{Fix}(\mathrm{z})|=\sum_{\mathrm{C} \in \mathscr{C} \ell(\mathfrak{g})} \delta_{\mathrm{C}, \mathrm{zC}}=\sum_{\mathrm{C} \in \mathscr{C} \ell(\mathfrak{g})} \frac{1}{\llbracket \mathrm{C} \rrbracket} \sum_{\alpha \in \mathfrak{g}} \alpha(\mathrm{zC}) \overline{\alpha(\mathrm{C})}= \\
& \sum_{\mathrm{C} \in \mathscr{C} \ell(\mathfrak{g})} \frac{1}{\llbracket \mathrm{C} \rrbracket} \sum_{\alpha \in \mathfrak{g}} \varpi_{\mathrm{z}}(\alpha)|\alpha(\mathrm{C})|^{2}=\sum_{\alpha \in \mathfrak{g}} \varpi_{\mathrm{z}}(\alpha) \sum_{\mathrm{C} \in \mathscr{C} \ell(\mathfrak{g})} \frac{|\alpha(\mathrm{C})|^{2}}{\llbracket \mathrm{C} \rrbracket}=\sum_{\alpha \in \mathfrak{g}} \varpi_{\mathrm{z}}(\alpha)
\end{aligned}
$$

leading to

$$
\left|\mathfrak{g}_{\xi}\right|=\sum_{\alpha \in \mathfrak{g}} \frac{1}{|Z|} \sum_{\mathrm{z} \in Z} \overline{\xi(\mathrm{z})} \varpi_{\mathrm{z}}(\alpha)=\frac{1}{|Z|} \sum_{\mathrm{z} \in Z} \overline{\xi(\mathrm{z})} \sum_{\alpha \in \mathfrak{g}} \varpi_{\mathrm{z}}(\alpha)=\frac{1}{|Z|} \sum_{\mathrm{z} \in Z} \overline{\xi(\mathrm{z})}|\mathrm{Fix}(\mathrm{z})| .
$$


To prove 2), note that

$$
\begin{aligned}
& \frac{\llbracket \mathfrak{g} \rrbracket}{\llbracket \mathfrak{g}_{\xi} \rrbracket}=\frac{\sum_{\alpha \in \mathfrak{g}_{\xi}} S_{0 \alpha}^{2}}{\sum_{\alpha \in \mathfrak{g}} S_{0 \alpha}^{2}}=\frac{1}{\llbracket \mathfrak{g}^{\perp} \rrbracket} \sum_{\alpha \in \mathfrak{g}} \mathrm{d}_{\alpha}^{2} \frac{1}{|Z|} \sum_{\mathrm{z} \in Z} \overline{\xi(\mathrm{z})} \varpi_{\mathrm{z}}(\alpha) \\
& =\frac{1}{|Z| \llbracket \mathfrak{g}^{\perp} \rrbracket} \sum_{\mathrm{z} \in Z} \overline{\xi(\mathrm{z})} \sum_{\alpha \in \mathfrak{g}} \alpha(\mathrm{z}) \mathrm{d}_{\alpha}=\frac{1}{|Z|} \sum_{\mathrm{z} \in Z} \overline{\xi(\mathrm{z})} \delta_{\mathrm{z}, \mathfrak{g}^{\perp}}=\frac{1}{|Z|}
\end{aligned}
$$

is independent of $\xi \in \hat{Z}$, where we have used Lemma 9. It follows that for all $\xi \in \hat{Z}$ one has $\llbracket \mathfrak{g}_{\xi} \rrbracket=\llbracket \mathfrak{g}_{\mathbf{1}} \rrbracket$, which is tantamount to $\mathfrak{g}_{\xi} \in \mathrm{Z}\left(\mathfrak{g}_{\mathbf{1}}^{\perp}\right)$. That $\mathfrak{g}_{\xi} \mathfrak{g}_{\eta}=\mathfrak{g}_{\xi \eta}$ for $\xi, \eta \in \hat{Z}$ can be seen as follows: $N_{\alpha \beta}^{\gamma}>0$ with $\alpha \in \mathfrak{g}_{\xi}$ and $\beta \in \mathfrak{g}_{\eta}$ implies that $\gamma \in \mathfrak{g}_{\xi} \mathfrak{g}_{\eta}$ by the generalized product rule Theorem 4 , and $\gamma \in \mathfrak{g}_{\xi \eta}$ by Lemma 16. But this means that the map $\xi \mapsto \mathfrak{g} \xi$ sets up an isomorphism $\hat{Z} \cong Z^{\perp}$, and because $\hat{Z} \cong Z$ by Pontryagin duality, we get that $Z \cong Z^{\perp}$. Finally, it is clear that

$$
\cup Z^{\perp}=\bigcup_{\xi \in \hat{Z}} \mathfrak{g}_{\xi}=\mathfrak{g}
$$

hence $\mathfrak{g}^{\perp}=\left(\cup Z^{\perp}\right)^{\perp}=(\mathfrak{g} / Z)^{\perp} / Z^{\perp}$ according to Eq. (5.2).

As to 3 ), notice that (since $\mathfrak{g}_{1} \subseteq \mathfrak{g}$ ) each $\mathfrak{g}_{1}$-class $\mathfrak{C} \in \mathscr{C} \ell\left(\mathfrak{g}_{1}\right)$ is a union of $\mathfrak{g}$-classes by Lemma 10, hence there exists some $\mathrm{C} \in \mathscr{C} l(\mathfrak{g})$ contained in $\mathfrak{C}$. But for $\mathrm{z} \in Z$ the restrictions of $\boldsymbol{\rho}_{\mathrm{C}}$ and $\boldsymbol{\rho}_{\mathrm{zC}}$ to $\mathfrak{g}_{1}$ coincide, consequently one has $Z \mathrm{C} \subseteq \mathfrak{C}$. To prove that this containment is actually an equality, observe that one has

$$
\begin{array}{r}
\sum_{\alpha \in \mathfrak{g}_{\xi}} \sum_{p \in Z \mathrm{C}}\left|S_{\alpha p}\right|^{2}=\frac{1}{\left|Z_{\mathrm{C}}\right|} \sum_{\mathrm{z} \in Z} \sum_{p \in \mathrm{ZC}} \sum_{\alpha \in \mathfrak{g}_{\xi}}\left|S_{\alpha p}\right|^{2}=\frac{1}{\left|Z_{\mathrm{C}}\right|} \sum_{\mathrm{z} \in Z \text { }} \sum_{\alpha \in \mathfrak{g}_{\xi}}|\alpha(\mathrm{zC})|^{2} \sum_{p \in \mathrm{ZC}} S_{0 p}^{2} \\
=\frac{\left[Z: Z_{\mathrm{C}}\right]}{\llbracket \mathrm{C} \rrbracket} \sum_{\alpha \in \mathfrak{g}_{\xi}}|\alpha(\mathrm{C})|^{2}=\frac{\left[Z: Z_{\mathrm{C}}\right]}{\llbracket \mathrm{C} \rrbracket} \sum_{\alpha \in \mathfrak{g}} \frac{1}{|Z|} \sum_{\mathrm{z} \in Z} \overline{\xi(\mathrm{z})} \varpi_{\mathrm{z}}(\alpha)|\alpha(\mathrm{C})|^{2} \\
=\frac{1}{\left|Z_{\mathrm{C}}\right|} \sum_{\mathrm{z} \in Z} \frac{\overline{\xi(\mathrm{z})}}{\llbracket \mathrm{C} \rrbracket} \sum_{\alpha \in \mathfrak{g}} \alpha(\mathrm{zC}) \overline{\alpha(\mathrm{C})}=\frac{1}{\left|Z_{\mathrm{C}}\right|} \sum_{\mathrm{z} \in Z_{\mathrm{C}}} \overline{\xi(\mathrm{z})}
\end{array}
$$

for $\xi \in \hat{Z}$, and in particular

$$
\sum_{\alpha \in \mathfrak{g}_{1}} \sum_{p \in \mathfrak{C} \backslash Z \mathbb{C}}\left|S_{\alpha p}\right|^{2}=\sum_{\alpha \in \mathfrak{g}_{1}} \sum_{p \in \mathfrak{C}}\left|S_{\alpha p}\right|^{2}-\sum_{\alpha \in \mathfrak{g}_{1}} \sum_{p \in Z \mathbb{C}}\left|S_{\alpha p}\right|^{2}=\left\langle\mathfrak{g}_{\mathbf{1}}, \mathfrak{C}\right\rangle-1=0
$$

because $\left\langle\mathfrak{g}_{1}, \mathfrak{C}\right\rangle=1$ for all $\mathfrak{C} \in \mathscr{C} \ell\left(\mathfrak{g}_{1}\right)$ according to Corollary 4 ; since $\mathfrak{g}_{1}$ contains the vacuum 0 , this can only happen if $\mathfrak{C} \backslash Z \mathrm{C}$ is empty, proving that indeed $\mathfrak{C}=Z \mathrm{C}$. Taking this into account, one has

$$
\frac{1}{\llbracket Z C \rrbracket}=\frac{1}{\left|Z_{\mathrm{C}}\right|} \sum_{\mathrm{z} \in Z} \sum_{p \in \mathrm{ZC}} S_{0 p}^{2}=\frac{1}{\left|Z_{\mathrm{C}}\right|} \sum_{\mathrm{z} \in Z} \llbracket z \mathrm{z} \rrbracket=\frac{\left[Z: Z_{\mathrm{C}}\right]}{\llbracket \mathrm{C} \rrbracket} .
$$

Finally,

$$
\left\langle\mathfrak{g}_{\xi}, Z \mathrm{C}\right\rangle=\sum_{\alpha \in \mathfrak{g}_{\xi}} \sum_{p \in Z \mathrm{C}}\left|S_{\alpha p}\right|^{2}= \begin{cases}1 & \text { if } Z_{\mathrm{C}}<\operatorname{ker} \xi \\ 0 & \text { otherwise }\end{cases}
$$

according to the above, proving the last assertion. 
Given an FC set $\mathfrak{g}$, it is natural to ask whether it is a central quotient of another FC set. This leads to the following notion.

Definition 10. Let $\mathfrak{g} \in \mathscr{L}$ denote an FC set and $A$ an Abelian group. An $A$-extension of $\mathfrak{g}$ is an FC set $\mathfrak{h} \in \mathscr{L}$ such that $\mathfrak{h} / Z=\mathfrak{g}$ for some central subgroup $Z<Z(\mathfrak{h})$ isomorphic to $A$.

Lemma 18. For an Abelian group $A$ and $\mathfrak{g} \in \mathscr{L}$, the different $A$-extensions of $\mathfrak{g}$ are in one-to-one correspondence with subgroups of $Z\left(\mathfrak{g}^{\perp}\right)$ isomorphic to $A$.

Proof. Suppose that $\mathfrak{h} \in \mathscr{L}$ is an $A$-extension of $\mathfrak{g}$, i.e. $\mathfrak{g}=\mathfrak{h} / Z$ for some subgroup $Z<\mathrm{Z}(\mathfrak{h})$ isomorphic to $A$. By 2) of Theorem $6 Z^{\perp}=\{\mathfrak{h} \xi \mid \xi \in \hat{Z}\}$ is a subgroup of $\mathrm{z}\left(\mathfrak{g}^{\perp}\right)$ isomorphic to $Z$, hence to $A$. Conversely, for any subgroup $Z<\mathrm{Z}\left(\mathfrak{g}^{\perp}\right)$ isomorphic to $A$, $\mathfrak{h}=\left(\mathfrak{g}^{\perp} / Z\right)^{\perp}$ is an FC set, and by 2) of Theorem 6 one has $\mathfrak{h} / Z^{\perp}=\left(\mathfrak{g}^{\perp} / Z\right)^{\perp} / Z^{\perp}=\mathfrak{g}$ for some subgroup $Z^{\perp}<Z(\mathfrak{h})$ isomorphic to $Z$, hence to $A$ as well, i.e. $\mathfrak{h}$ is an $A$-extension of $\mathfrak{g}$.

Corollary 10. Every $F C$ set $\mathfrak{g} \in \mathscr{L}$ has a maximal central extension, the dual of the maximal central quotient of $\mathfrak{g}^{\perp}$.

Definition 11. An FC set $\mathfrak{g} \in \mathscr{L}$ is nilpotent if it can be obtained from the trivial FC set by a sequence of central extensions.

The rationale of this terminology is that if $\mathfrak{g} \in \mathscr{L}$ is local, hence the associated algebra $\mathcal{V}_{\mathfrak{g}}$ is isomorphic to the character ring of some finite group $G$, the FC set $\mathfrak{g}$ is nilpotent according to the above definition precisely when $G$ is nilpotent.

Lemma 19. If $\mathfrak{g} \in \mathscr{L}$ is nilpotent, then $\llbracket \mathfrak{g}^{\perp} \rrbracket \in \mathbb{Z}$.

Proof. According to Theorem 6 , if $\mathfrak{g} \in \mathscr{L}$ is a central extension of $\mathfrak{h} \in \mathscr{L}$, then $\llbracket \mathfrak{g}^{\perp} \rrbracket$ is an integer multiple of $\llbracket \mathfrak{h}^{\perp} \rrbracket$. The claim follows by induction.

We will see in Corollary 13 that $\llbracket \mathfrak{g}^{\perp} \rrbracket \in \mathbb{Z}$ implies that the quantum dimension of any element of $\mathfrak{g}$ is either an integer or the square root of an integer. That the latter possibility can occur is exemplified by the maximal FC set of the Ising model (the minimal Virasoro model of central charge $\frac{1}{2}$ ), which is nilpotent while having a primary of dimension $\sqrt{2}$. We conjecture that many results about (finite) nilpotent groups carry over to nilpotent FC sets, like the following property, which is known to be equivalent to nilpotency for finite groups.

Conjecture 2. If $\mathfrak{g}$ is nilpotent and $d$ is an integer dividing $\llbracket \mathfrak{g}^{\perp} \rrbracket$, then there exists an FC set $\mathfrak{h} \subseteq \mathfrak{g}$ such that $\llbracket \mathfrak{h}^{\perp} \rrbracket=d$.

\section{The Galois Action}

Let us briefly recall the basics of the Galois action in RCFT [5,7,10]. It is known that, denoting by $N$ the least common multiple of the denominators of the conformal weights $\mathrm{h}_{p}$, the field obtained by adjoining to the rationals $\mathbb{Q}$ the quantum dimensions $\mathrm{d}_{p}$ and the exponentiated conformal weights $\omega(p)$ is the cyclotomic field $\mathbb{Q}\left[\zeta_{N}\right]$ of conductor $N$, generated by a primitive root of unity $\zeta_{N}=e^{\frac{2 \pi i}{N}}$. The Galois group of $\mathbb{Q}\left[\zeta_{N}\right]$ is isomorphic to the group $(\mathbb{Z} / N \mathbb{Z})^{\times}$of prime residues $\bmod N$, with each $\ell \in(\mathbb{Z} / N \mathbb{Z})^{\times}$ corresponding to a Galois transformation $\sigma_{\ell}$ mapping $\zeta_{N}$ to $\zeta_{N}^{\ell}$ (while leaving all rationals 
fixed). Since the conformal weights of primaries are rational numbers, one has $\sigma_{\ell} \circ \omega=\omega^{\ell}$ for the exponentiated conformal weights.

Because fusion matrices have rational integer matrix elements, the irreducible representations of the Verlinde algebra $\mathcal{V}$ (and of its subalgebras) are permuted between themselves by the Galois transformations $\sigma_{\ell}$. In other words, for each $\ell \in(\mathbb{Z} / N \mathbb{Z})^{\times}$there exists a permutation $\pi(\ell): p \mapsto \ell p$ of the primaries such that

$$
\sigma_{\ell} \circ \boldsymbol{\rho}_{p}=\boldsymbol{\rho}_{\ell p}
$$

and the mapping $\pi: \ell \mapsto \pi(\ell)$ is clearly a homomorphism. Furthermore,

$$
\sigma_{\ell}\left(\mathrm{d}_{p}\right)=\epsilon_{\ell}(p) \frac{\mathrm{d}_{\ell p}}{\mathrm{~d}_{\ell 0}}
$$

where $\epsilon_{\ell}(p)= \pm 1$ depending on the sign of $\sigma_{\ell}\left(\alpha_{p}\right)$. Note that it follows from Eqs. (2.7) and (6.1) that $\left|\sigma_{\ell}\left(\mathrm{d}_{p}\right)\right|=\left|\boldsymbol{\rho}_{\ell 0}(p)\right| \leq \mathrm{d}_{p}$, i.e. $\mathrm{d}_{\ell p} \leq \mathrm{d}_{p} \mathrm{~d}_{\ell 0}$. Finally, as a consequence of the fact that the modular representation has as kernel a congruence subgroup of level $N$, one has for all primaries $p$ and any $\ell \in(\mathbb{Z} / N \mathbb{Z})^{\times}$

$$
\mathrm{h}_{\ell p}-\mathrm{h}_{\ell 0} \in \ell^{2} \mathrm{~h}_{p}+\mathbb{Z}
$$

Lemma 20. For $\ell \in(\mathbb{Z} / N \mathbb{Z})^{\times}$let $\boldsymbol{\Theta}_{\ell}=\left\{p \mid \sigma_{\ell}\left(d_{p}\right)=\boldsymbol{\epsilon}_{\ell}(p) d_{p}\right\}$. Then $p, q \in \boldsymbol{\Theta}_{\ell}$ and $N_{p q}^{r}>0$ implies $r \in \boldsymbol{\Theta}_{\ell}$ and $\boldsymbol{\epsilon}_{\ell}(r)=\boldsymbol{\epsilon}_{\ell}(p) \epsilon_{\ell}(q)$. As a consequence, both $\boldsymbol{\Theta}_{\ell}$ and $\boldsymbol{\Theta}_{\ell}^{+}=\left\{p \mid \sigma_{\ell}\left(d_{p}\right)=d_{p}\right\}$ are FC sets.

Proof. Applying $\sigma_{\ell}$ to both sides of $\sum_{r} N_{p q}^{r} \mathrm{~d}_{r}=\mathrm{d}_{p} \mathrm{~d}_{q}$ gives

$$
\boldsymbol{\epsilon}_{\ell}(p) \boldsymbol{\epsilon}_{\ell}(q) \sum_{r} N_{p q}^{r} \sigma_{\ell}\left(\mathrm{d}_{r}\right)=\epsilon_{\ell}(p) \epsilon_{\ell}(q) \sigma_{\ell}\left(\mathrm{d}_{p} \mathrm{~d}_{q}\right)=\mathrm{d}_{p} \mathrm{~d}_{q}=\sum_{r} N_{p q}^{r} \mathrm{~d}_{r}
$$

for $p, q \in \boldsymbol{\Theta}_{\ell}$. After rearrangement and taking real parts, one gets

$$
\sum_{r} N_{p q}^{r} \operatorname{Re}\left\{\mathrm{d}_{r}-\epsilon_{\ell}(p) \epsilon_{\ell}(q) \sigma_{\ell}\left(\mathrm{d}_{r}\right)\right\}=0 .
$$

Because $\left|\sigma_{\ell}\left(d_{r}\right)\right| \leq d_{r}$, and the real part of a complex number cannot exceed its modulus, we conclude that all terms on the left-hand side are non-negative, hence they should all vanish, that is $N_{p q}^{r}=0$ unless $\sigma_{\ell}\left(\mathrm{d}_{r}\right)=\epsilon_{\ell}(p) \epsilon_{\ell}(q) \mathrm{d}_{r}=\epsilon_{\ell}(r) \mathrm{d}_{r}$. Since $\boldsymbol{\Theta}_{\ell}^{+}=$ $\left\{p \in \boldsymbol{\Theta}_{\ell} \mid \epsilon_{\ell}(p)=1\right\}$, the assertion follows.

Remark 4. Note that, as a consequence of $\epsilon_{\ell}(p)= \pm 1$, the FC set $\boldsymbol{\Theta}_{\ell}$ either coincides with $\Theta_{\ell}^{+}$or is a $\mathbb{Z}_{2}$-extension of it.

Corollary 11. Both $\boldsymbol{\Theta}^{+}=\left\{p \mid d_{p} \in \mathbb{Z}\right\}$ and $\boldsymbol{\Theta}=\left\{p \mid d_{p}^{2} \in \mathbb{Z}\right\}$ are FC sets.

Proof. Because quantum dimensions are algebraic integers, one has $p \in \Theta^{+}$iff $\mathrm{d}_{p} \in \mathbb{Q}$, i.e. $\sigma_{\ell}\left(\mathrm{d}_{p}\right)=\mathrm{d}_{p}$ for all $\ell \in(\mathbb{Z} / N \mathbb{Z})^{\times}$, hence

$$
\boldsymbol{\Theta}^{+}=\bigcap_{\ell \in(\mathbb{Z} / N \mathbb{Z})^{\times}} \boldsymbol{\Theta}_{\ell}^{+} .
$$

This implies at once $\boldsymbol{\Theta}^{+} \in \mathscr{L}$ by Lemma 20. A similar argument works for $\boldsymbol{\Theta}$, exploiting the fact that $\epsilon_{\ell}(p)= \pm 1$. 
From now on, we shall consider a fixed FC set $\mathfrak{g} \in \mathscr{L}$.

Proposition 7. The Galois permutations $\pi(\ell)$ map $\mathfrak{g}$-classes to $\mathfrak{g}$-classes, i.e. $\ell C=$ $\{\ell p \mid p \in C\} \in \mathscr{C} \ell(\mathfrak{g})$ for every class $C \in \mathscr{C} \ell(\mathfrak{g})$, in such a way that $\ell(z C)=z^{\ell}(\ell C)$ for $z \in Z(\mathfrak{g})$. Moreover, overlaps are left invariant, $\langle\mathfrak{b}, \ell \mathcal{C}\rangle=\langle\mathfrak{b}, C\rangle$ for all $\mathfrak{b} \in \mathcal{B} \ell(\mathfrak{g})$, while $\llbracket \ell C \rrbracket=\sigma_{\ell}(\llbracket C \rrbracket)$.

Proof. That $\ell \mathrm{C} \in \mathscr{C} \ell(\mathfrak{g})$ follows at once from Eq. (6.1), while for $\mathrm{z} \in \mathrm{Z}(\mathfrak{g})$

$$
\begin{aligned}
& \alpha(\ell(\mathrm{zC}))=\sigma_{\ell}(\alpha(\mathrm{zC}))=\sigma_{\ell}\left(\varpi_{\mathrm{z}}(\alpha) \alpha(\mathrm{C})\right)= \\
& \quad=\varpi_{\mathrm{z}}(\alpha)^{\ell} \sigma_{\ell}(\alpha(\mathrm{C}))=\varpi_{\mathrm{z}^{\ell}}(\alpha) \alpha(\ell \mathrm{C})=\alpha\left(\mathrm{z}^{\ell}(\ell \mathrm{C})\right)
\end{aligned}
$$

by Eqs. (6.1) and (4.2), since $\varpi_{z}(\alpha)$ is a root of unity. As to the rest,

remember that the overlap $\langle\mathfrak{b}, C\rangle$ is the multiplicity of the irrep $\boldsymbol{\rho}_{\mathrm{C}}$ in the irreducible decomposition of the representation $\Delta_{\mathfrak{b}}$ associated to the block $\mathfrak{b}$. Since $\Delta_{\mathfrak{b}}$ is integral, it equals all its Galois conjugates, hence it contains the irreps $\boldsymbol{\rho}_{\ell \mathrm{C}}=\sigma_{\ell} \circ \boldsymbol{\rho}_{\mathrm{C}}$ and $\boldsymbol{\rho}_{\mathrm{C}}$ with the same multiplicity, i.e. $\langle\mathfrak{b}, \ell \mathrm{C}\rangle=\langle\mathfrak{b}, \mathrm{C}\rangle$. Finally,

$$
\sigma_{\ell}(\llbracket \mathrm{C} \rrbracket)=\sum_{\alpha \in \mathfrak{g}} \sigma_{\ell}\left(|\alpha(\mathrm{C})|^{2}\right)=\sum_{\alpha \in \mathfrak{g}}|\alpha(\ell \mathrm{C})|^{2}=\llbracket \ell \mathrm{C} \rrbracket
$$

by Eq. (3.7), proving the assertion.

Corollary 12. The Galois permutations $\pi(\ell)$ map $\mathfrak{g}$-blocks to $\mathfrak{g}$-blocks, i.e. $\ell \mathfrak{b}=\{\ell p \mid p \in \mathfrak{b}\}$ $! \in \mathcal{B} \ell(\mathfrak{g})$ for any block $\mathfrak{b} \in \mathcal{B} \ell(\mathfrak{g})$, in such a way that $\llbracket \ell \mathfrak{b} \rrbracket=\sigma_{\ell}(\llbracket \mathfrak{b} \rrbracket)$ and $\langle\ell \mathfrak{b}, C\rangle=\langle\mathfrak{b}, C\rangle$ for all $C \in \mathscr{C l}(\mathfrak{g})$. As a consequence, every $F C$ set $\mathfrak{g} \in \mathscr{L}$ is self-conjugate, i.e.the charge conjugate $\bar{\alpha}$ of any primary $\alpha \in \mathfrak{g}$ also belongs to $\mathfrak{g}$.

Proof. The first claim follows from Proposition 7 by duality. As to the second, note that $\sigma_{-1}$ is complex conjugation, hence $\pi(-1)$ is charge conjugation. Because $\pi(-1)$ leaves the vacuum $0 \in \mathfrak{g}$ invariant, it should map $\mathfrak{g}$ onto itself.

Lemma 21. $\mathfrak{g} \subseteq \boldsymbol{\Theta}_{\ell}^{+}$iff $\ell \mathfrak{g}^{\perp}=\mathfrak{g}^{\perp}$.

Proof. Since $\mathrm{d}_{\alpha}=\boldsymbol{\rho}_{\mathfrak{g}^{\perp}}(\alpha)$, one has $\boldsymbol{\rho}_{\ell \mathfrak{g}^{\perp}}(\alpha)=\left(\sigma_{\ell} \circ \boldsymbol{\rho}_{\mathfrak{g}^{\perp}}\right)(\alpha)=\sigma_{\ell}\left(\mathrm{d}_{\alpha}\right)$ for $\alpha \in \mathfrak{g}$, hence $\ell \mathfrak{g}^{\perp}=\mathfrak{g}^{\perp}$ exactly when $\sigma_{\ell}\left(\mathrm{d}_{\alpha}\right)=\mathrm{d}_{\alpha}$ for all $\alpha \in \mathfrak{g}$.

Lemma 22. If $\mathfrak{g} \subseteq \boldsymbol{\Theta}_{\ell}^{+}$and $\mathfrak{b} \in \mathcal{B} \ell(\mathfrak{g})$, then the ratio $\frac{\sigma_{\ell}\left(d_{p}\right)}{d_{p}}$ is independent of $p \in \mathfrak{b}$.

Proof. Let $\mathfrak{D}$ denote the vector whose components are the quantum dimensions $\mathrm{d}_{p}$ for $p \in \mathfrak{b}$. Eq. (2.6) implies that $\mathrm{N}_{\mathfrak{b}}(\alpha) \mathfrak{D}=\mathrm{d}_{\alpha} \mathfrak{D}$ for all $\alpha \in \mathfrak{g}$, and applying $\sigma_{\ell}$ to both sides gives $\mathrm{N}_{\mathfrak{b}}(\alpha) \sigma_{\ell}(\mathfrak{D})=\mathrm{d}_{\alpha} \sigma_{\ell}(\mathfrak{D})$, taking into account that the $\mathrm{N}_{\mathfrak{b}}(\alpha)$ are integer matrices and $\sigma_{\ell}\left(\mathrm{d}_{\alpha}\right)=\mathrm{d}_{\alpha}$. But this means that both $\mathfrak{D}$ and $\sigma_{\ell}(\mathfrak{D})$ belong to the common eigenspace of the matrices $N_{\mathfrak{b}}(\alpha)$ corresponding to the irrep $\boldsymbol{\rho}_{\mathfrak{g}} \perp$ of $\mathcal{V}_{\mathfrak{g}}$, and because $\left\langle\mathfrak{b}, \mathfrak{g}^{\perp}\right\rangle=1$ by Corollary 4 , this eigenspace has dimension 1 , hence $\sigma_{\ell}(\mathfrak{D})$ and $\mathfrak{D}$ are proportional to each other.

It follows from Corollary 11 that $\mathscr{L}_{\text {int }}^{+}=\left\{\mathfrak{g} \subseteq \mathscr{L} \mid \mathfrak{g} \subseteq \boldsymbol{\Theta}^{+}\right\}$is a sublattice of $\mathscr{L}$ consisting of those FC sets all of whose elements have integer quantum dimension. Such FC sets have special properties, as exemplified by the following result. 
Lemma 23. If $\mathfrak{g} \in \mathscr{L}_{i n t}^{+}$, then for every block $\mathfrak{b} \in \mathcal{B} \ell(\mathfrak{g})$ there exists an algebraic integer $d_{\mathfrak{b}}$ such that the quantum dimension of the primaries contained in $\mathfrak{b}$ are rational multiples of $d_{\mathfrak{b}}$.

Proof. Using the notation $\mathrm{d}_{\mathfrak{b}}=\min \left\{\mathrm{d}_{p} \mid p \in \mathfrak{b}\right\}$, one has

$$
\sigma_{\ell}\left(\frac{\mathrm{d}_{p}}{\mathrm{~d}_{\mathfrak{b}}}\right)=\frac{\mathrm{d}_{p}}{\mathrm{~d}_{\mathfrak{b}}}
$$

for all $\ell \in(\mathbb{Z} / N \mathbb{Z})^{\times}$by Lemma 22 . Since $d_{\mathfrak{b}}$ is an algebraic integer, and an algebraic number fixed by all $\sigma_{\ell}$ is rational, the result follows.

Proposition 8. The following statements are equivalent:

(a) $\mathfrak{g} \subseteq \boldsymbol{\Theta}_{\ell}$;

(b) $\sigma_{\ell}\left(d_{\alpha}^{2}\right)=d_{\alpha}^{2}$ for all $\alpha \in \mathfrak{g}$;

(c) $\sigma_{\ell}\left(\llbracket \mathfrak{g}^{\perp} \rrbracket\right)=\llbracket \mathfrak{g}^{\perp} \rrbracket$;

(d) $\ell \mathfrak{g}^{\perp} \in Z(\mathfrak{g})$.

Proof. For one thing, $\mathfrak{g} \subseteq \boldsymbol{\Theta}_{\ell}$, i.e. $\sigma_{\ell}\left(\mathrm{d}_{\alpha}\right)=\epsilon_{\ell}(\alpha) \mathrm{d}_{\alpha}= \pm \mathrm{d}_{\alpha}$ for all $\alpha \in \mathfrak{g}$ implies $\sigma_{\ell}\left(\mathrm{d}_{\alpha}^{2}\right)=$ $\mathrm{d}_{\alpha}^{2}$, which in turn implies $\sigma_{\ell}\left(\llbracket \mathfrak{g}^{\perp} \rrbracket\right)=\llbracket \mathfrak{g}^{\perp} \rrbracket$ by Eq. (3.11). But $\llbracket \mathfrak{g}^{\perp} \rrbracket=\sigma_{\ell}\left(\llbracket \mathfrak{g}^{\perp} \rrbracket\right)=\llbracket \ell \mathfrak{g}^{\perp} \rrbracket$ gives at once $\ell \mathfrak{g}^{\perp} \in \mathrm{Z}(\mathfrak{g})$ and

$$
\varpi_{\ell \mathfrak{g}^{\perp}}(\alpha) \mathrm{d}_{\alpha}=\boldsymbol{\rho}_{\ell \mathfrak{g}^{\perp}}(\alpha)=\left(\sigma_{\ell} \circ \boldsymbol{\rho}_{\mathfrak{g}^{\perp}}\right)(\alpha)=\sigma_{\ell}\left(\mathrm{d}_{\alpha}\right)=\boldsymbol{\epsilon}_{\ell}(\alpha) \frac{\mathrm{d}_{\ell \alpha}}{\mathrm{d}_{\ell 0}}
$$

for $\alpha \in \mathfrak{g}$, as a consequence of Eq. (6.2). Since quantum dimensions are positive numbers while $\varpi_{\ell \mathfrak{g}^{\perp}}(\alpha)$ has unit modulus, one concludes that $\varpi_{\ell \mathfrak{g}^{\perp}}(\alpha)=\boldsymbol{\epsilon}_{\ell}(\alpha)$ and $\mathfrak{g} \subseteq \boldsymbol{\Theta}_{\ell}$, completing the proof.

Corollary 13. $\llbracket \mathfrak{g}^{\perp} \rrbracket \in \mathbb{Z}$ iff $\mathfrak{g} \subseteq \boldsymbol{\Theta}$.

Proof. By Proposition $8, \llbracket \mathfrak{g}^{\perp} \rrbracket \in \mathbb{Z}$ iff $\mathfrak{g} \subseteq \boldsymbol{\Theta}_{\ell}$ for all $\ell \in(\mathbb{Z} / N \mathbb{Z})^{\times}$, i.e. $\mathfrak{g} \subseteq \boldsymbol{\Theta}$.

By the above, $\mathscr{L}_{\text {int }}=\left\{\mathfrak{g} \in \mathscr{L} \mid \llbracket \mathfrak{g}^{\perp} \rrbracket \in \mathbb{Z}\right\}$ is a sublattice of $\mathscr{L}$ containing $\mathscr{L}_{\text {int }}^{+}$. Note that, according to Lemma 19 , every nilpotent FC set belongs to $\mathscr{L}_{\text {int }}$, but the converse need not be true.

Lemma 24. If $\mathfrak{g} \subseteq \boldsymbol{\Theta}_{\ell}$ and $\mathfrak{b} \in \mathcal{B} \ell(\mathfrak{g})$, then for all $p \in \mathfrak{b}$

$$
\frac{d_{\ell p}}{d_{p}}=\sqrt{\frac{\llbracket \mathfrak{b} \rrbracket}{\llbracket \ell \mathfrak{b} \rrbracket}}
$$

Proof. If $\mathfrak{g} \subseteq \mathbf{\Theta}_{\ell}^{+}$, then it follows from Eq. (6.2) and Lemma 22 that

$$
\frac{S_{0 \ell p}^{2}}{S_{0 p}^{2}}=\left(\frac{\mathrm{d}_{\ell p}}{\mathrm{~d}_{p}}\right)^{2}=\left(\frac{\sigma_{\ell}\left(\mathrm{d}_{p}\right)}{\mathrm{d}_{p}}\right)^{2} \epsilon_{\ell}(p)^{2} \mathrm{~d}_{\ell 0}^{2}=A
$$

is independent of $p \in \mathfrak{b}$, hence

$$
\llbracket \mathfrak{b} \rrbracket=\sum_{p \in \mathfrak{b}} \frac{1}{S_{0 p}^{2}}=A \sum_{p \in \mathfrak{b}} \frac{1}{S_{0 \ell p}^{2}}=A \llbracket \ell \mathfrak{b} \rrbracket
$$


from which one concludes

$$
\left(\frac{\mathrm{d}_{\ell p}}{\mathrm{~d}_{p}}\right)^{2}=\frac{\llbracket \mathfrak{b} \rrbracket}{\llbracket \ell \mathfrak{b} \rrbracket}
$$

implying Eq.(6.4), since quantum dimensions are positive numbers.

If $\mathfrak{g} \subseteq \boldsymbol{\Theta}_{\ell}$ is not contained in $\boldsymbol{\Theta}_{\ell}^{+}$, then $\mathfrak{g}$ is a $\mathbb{Z}_{2}$-extension of $\mathfrak{g}_{+}=\mathfrak{g} \cap \boldsymbol{\Theta}_{\ell}^{+} \subseteq \boldsymbol{\Theta}_{\ell}^{+}$, hence any block $\mathfrak{b} \in \mathcal{B} \ell(\mathfrak{g})$ is either itself a $\mathfrak{g}_{+}$-block, or $\mathfrak{b}=\mathfrak{b}_{+} \cup \mathfrak{b}_{-}$with $\mathfrak{b}_{ \pm} \in \mathcal{B} \ell\left(\mathfrak{g}_{+}\right)$ and $\llbracket \mathfrak{b}_{ \pm} \rrbracket=2 \llbracket \mathfrak{b} \rrbracket$ (cf. Theorem 6). In either case Eq. (6.4) follows by the above argument, since $\ell \mathfrak{b}=\ell \mathfrak{b}_{+} \cup \ell \mathfrak{b}_{-}$and $\llbracket \ell \mathfrak{b}_{ \pm} \rrbracket=\llbracket \mathfrak{b}_{ \pm} \rrbracket=2 \llbracket \mathfrak{b} \rrbracket=2 \llbracket \ell \mathfrak{b} \rrbracket$ by Corollary 12 .

Lemma 24 gives a fairly precise description of the distribution of quantum dimensions (counted with multiplicity) in blocks related by Galois permutations, and a similar result for classes would be most desirable. Supported by extensive computational evidence, the following seems to hold.

Conjecture 3. If $\mathfrak{g} \subseteq \boldsymbol{\Theta}_{\ell}$ and $C \in \mathscr{C} \ell(\mathfrak{g})$ is a $\mathfrak{g}$-class, then the distribution of quantum dimensions (counted with multiplicity) is the same in $C$ and $\ell C$. Put differently, the univariate polynomial

$$
\mathcal{P}_{C}(x)=\prod_{p \in C}\left(x-S_{0 p}^{-2}\right)
$$

satisfies $\mathcal{P}_{\ell C}(x)=\mathcal{P}_{C}(x)$.

Remark 5. Note that, as a consequence of Lemma 10, it would be enough to prove Conjecture 3 for $\mathfrak{g}=\boldsymbol{\Theta}_{\ell}$, since this would imply the general case. Moreover, since $S_{0 p}^{-2}$ is an algebraic integer for each primary $p$, and because $\sigma_{\ell}\left(\mathcal{P}_{\mathrm{C}}(x)\right)=\mathcal{P}_{\ell \mathrm{C}}(x)$, the truth of Conjecture 3 would imply that all the coefficients of $\mathcal{P}_{\mathrm{C}}(x)$ are rational integers for $\mathfrak{g} \in \mathscr{L}_{\text {int }}$. But $\llbracket C \rrbracket \in \mathbb{Z}$ in case $\mathcal{P}_{\mathrm{C}}(x) \in \mathbb{Z}[x]$ : this would lead, in conjunction with Conjecture 1 , to the conclusion that $\llbracket C \rrbracket$ is an integer divisor of $\llbracket \mathfrak{g}^{\perp} \rrbracket$.

Finally, let's note that Eq. (6.5) should be contrasted with the following consequence of Lemma 24 for $\mathfrak{g}^{\perp} \subseteq \boldsymbol{\Theta}_{\ell}$ (with $\mathfrak{g}$-classes viewed as $\mathfrak{g}^{\perp}$-blocks):

$$
\mathcal{P}_{\ell C}(x)=\left(\frac{\llbracket \ell C \rrbracket}{\llbracket C \rrbracket}\right)^{|C|} \mathcal{P}_{C}\left(\frac{\llbracket C \rrbracket}{\llbracket \ell C \rrbracket} x\right) .
$$

\section{Local Sets and Twisters}

Remember that the FC set $\mathfrak{g} \in \mathscr{L}$ is local if $\mathfrak{g} \subseteq \mathfrak{g}^{\perp}$. We will denote by $\mathscr{L}_{\text {loc }}$ the set of local FC sets; note that, while the intersection of local FC sets is clearly local, this is not necessarily the case for their join, i.e. $\mathscr{L}_{1 \text { oc }}$ is generally not a sublattice of $\mathscr{L}$, because it may have several maximal elements. Actually, $\mathscr{L}_{\text {loc }}$ is itself a lattice precisely when it has a unique maximal element.

Lemma 25. $\mathfrak{g} \in \mathscr{L}$ is local iff each $\mathfrak{g}$-class is a union of $\mathfrak{g}$-blocks, or equivalently, each $\mathfrak{g}$-block is contained in a well-defined $\mathfrak{g}$-class.

Proof. This is a direct consequence of Lemma 10, keeping in mind that $\mathfrak{g}$-blocks are nothing but $\mathfrak{g}^{\perp}$-classes. 
From the point of view of orbifold deconstruction [3,6], this is the basic property of local FC sets. The point is that the vacuum block of an orbifold model (the set of primaries originating in the vacuum primary) is an FC set whose classes correspond to the different twisted sectors, i.e. collections of twisted modules with twist elements belonging to the same conjugacy class, while its blocks correspond to orbits of twisted modules. Since the conjugacy class of a twist element is the same for all twisted modules on the same orbit, every block should be included in a well-defined class, hence the vacuum block should be a local FC set by the above.

Lemma 26. If $\mathfrak{g} \in \mathscr{L}_{\text {IoC }}$ and $\mathcal{B} \ell_{C}(\mathfrak{g})=\{\mathfrak{b} \in \mathcal{B} \ell(\mathfrak{g}) \mid \mathfrak{b} \subseteq C\}$ denotes the set of $\mathfrak{g}$-blocks contained in the class $C \in \mathscr{C l}(\mathfrak{g})$, then

$$
\left|\mathcal{B} \ell_{C}(\mathfrak{g})\right|=\sum_{\mathfrak{b} \subseteq C}\left\langle\mathfrak{b}, \mathfrak{g}^{\perp}\right\rangle=\sum_{\mathfrak{b} \subseteq \mathfrak{g}^{\perp}}\langle\mathfrak{b}, C\rangle .
$$

Proof. The first equality follows from Corollary 4, while the second one from Eq. (3.19) with $\mathfrak{h}=\mathfrak{b}=\mathfrak{g}^{\perp}$.

Lemma 27. $\mathfrak{g} \in \mathscr{L}$ is local iff $\omega(\gamma)=\omega(\alpha) \omega(\beta)$ for all $\alpha, \beta, \gamma \in \mathfrak{g}$ such that $N_{\alpha \beta}^{\gamma}>0$.

Proof. This follows from the containment $\mathfrak{g} \subseteq \mathfrak{g}^{\perp}$ and Corollary 9.

Corollary 14. If the FC set $\mathfrak{g}$ is local, then $h_{\alpha} \in \frac{1}{2} \mathbb{Z}$ for $\alpha \in \mathfrak{g}$.

Proof. According to Corollary 12, $\alpha \in \mathfrak{g}$ implies $\bar{\alpha} \in \mathfrak{g}$. Since $N_{\alpha \bar{\alpha}}^{0}=1$ and $\omega(\bar{\alpha})=\omega(\alpha)$, Lemma 27 implies that $\omega(\alpha)^{2}=\omega(0)=1$, i.e. $\mathrm{h}_{\alpha} \in \frac{1}{2} \mathbb{Z}$ for $\alpha \in \mathfrak{g}$.

Note that the converse is not true: there are many FC sets in which all conformal weights belong to $\frac{1}{2} \mathbb{Z}$, but are nevertheless not local. On the other hand, the integrality of conformal weights implies locality by Lemma 27 , leading to the following notion $[3,6]$.

Definition 12. A twister is an FC set all of whose elements have integer conformal weight.

Lemma 28. Every local FC set $\mathfrak{g} \in \mathscr{L}_{\text {Ioc }}$ has a central class $\mathrm{R} \in Z(\mathfrak{g})$ such that $\varpi_{\mathrm{R}}(\alpha)=\omega(\alpha)$ for $\alpha \in \mathfrak{g}$.

Proof. By Lemma 27

$$
\sum_{\gamma \in \mathfrak{g}} N_{\alpha \beta}^{\gamma} \omega(\gamma) \mathrm{d}_{\gamma}=\sum_{\gamma \in \mathfrak{g}} N_{\alpha \beta}^{\gamma} \omega(\alpha) \omega(\beta) \mathrm{d}_{\gamma}=\omega(\alpha) \omega(\beta) \mathrm{d}_{\alpha} \mathrm{d}_{\beta}
$$

in case $\alpha, \beta \in \mathfrak{g}$, i.e. the map $\alpha \mapsto \omega(\alpha) \mathrm{d}_{\alpha}$ is an irrep of $\mathcal{V}_{\mathfrak{g}}$, hence there does exist a class $\mathrm{R} \in \mathscr{C} \ell(\mathfrak{g})$ such that $\boldsymbol{\rho}_{\mathrm{R}}(\alpha)=\omega(\alpha) \mathrm{d}_{\alpha}$. Since, according to Corollary $14, \omega(\alpha)=$ \pm 1 for elements of a local FC set, Lemma 15 implies that the class $\mathrm{R}$ is central with $\varpi_{\mathrm{R}}(\alpha)=\omega(\alpha)$.

Lemma 29. $\mathrm{R}^{2}=\mathfrak{g}^{\perp}$ for $\mathfrak{g} \in \mathscr{L}_{\text {IoC }}$, and $\mathfrak{g}$ is a twister iff $\mathrm{R}=\mathfrak{g}^{\perp}$.

Proof. $\varpi_{\mathrm{R}}(\alpha)=\omega(\alpha)$ for $\alpha \in \mathfrak{g}$ by Lemma 28 , hence $\varpi_{\mathrm{R}^{2}}(\alpha)=\omega(\alpha)^{2}=1$ because of Corollary 14 , proving that indeed $R^{2}=\mathfrak{g}^{\perp}$. On the other hand, $\mathrm{R}=\mathfrak{g}^{\perp}$ precisely when $\omega(\alpha)=\varpi_{\mathrm{R}}(\alpha)=1$ for all $\alpha \in \mathfrak{g}$, i.e. when $\mathfrak{g}$ is a twister. 
Corollary 15. Every local $F C$ set $\mathfrak{g} \in \mathscr{L}_{\text {loc }}$ is either a twister or a $\mathbb{Z}_{2}$-extension of a twister.

Proof. If $\mathfrak{g} \in \mathscr{L}_{1 \text { oc }}$ is not a twister, then the class $\mathrm{R}$ generates a central subgroup of order 2 , and the corresponding central quotient $\{\alpha \in \mathfrak{g} \mid \omega(\alpha)=1\}$ is clearly a twister, proving the assertion.

We shall call the class $R \in Z(\mathfrak{g})$, whose existence is guaranteed by Lemma 28 , the Ramond class of the local FC set $\mathfrak{g} \in \mathscr{L}_{\text {loc }}$. The rationale for this nomenclature is that, in case $\mathrm{R}$ differs from the trivial class $\mathfrak{g}^{\perp}$, there exists a suitable fermionic generalization of orbifold deconstruction in which the blocks contained in the trivial class account for the Neveu-Schwarz (bosonic) sector of the deconstructed model, while those in the Ramond class describe the fermionic (Ramond) sector.

Lemma 30. If $\mathfrak{g} \in \mathscr{L}_{10 C}$ and $\mathfrak{b} \in \mathcal{B} \ell(\mathfrak{g})$ is a block contained in the central class $z \in Z(\mathfrak{g})$, then the conformal weights of its elements multiplied by the order of the product $z \mathrm{R}$ differ by integers.

Proof. Let $n$ denote the order of the class $z \mathrm{R}$ viewed as an element of the Abelian group $\mathrm{Z}(\mathfrak{g})$. According to Lemma 8, for any two primaries $p$ and $q$ contained in the block $\mathfrak{b} \subseteq \mathrm{z}$ there exists $\alpha \in \mathfrak{g}$ such that $N_{\alpha p}^{q}>0$. By Lemma 17 this implies that $\omega(q)=\omega(\alpha) \omega(p) \varpi_{\mathrm{z}}(\alpha)^{-1}=\omega(p) \varpi_{\mathrm{z} \mathrm{R}}(\alpha)^{-1}$, and because $\varpi_{\mathrm{z} R}(\alpha)^{n}=\varpi_{\mathfrak{g}^{\perp}}(\alpha)=1$, this means that $\omega(q)^{n}=\omega(p)^{n}$, i.e. $n\left(\mathrm{~h}_{q}-\mathrm{h}_{p}\right) \in \mathbb{Z}$.

Corollary 16. A block is contained in the Ramond class $\mathrm{R}$ precisely when the conformal weights of its elements differ by integers.

We note that the above results can be generalized further: there is an intrinsic way to define Adams operations and power maps for local FC sets $[3,6]$, which lead to a welldefined notion of the order of classes, and for central classes this coincides with their multiplicative order (as elements of the center).

Lemma 31. If $C \in \mathscr{C l}(\mathfrak{g})$ is a class of a local $F C$ set $\mathfrak{g} \in \mathscr{L}_{1 \circ C}$, then $\langle\mathfrak{b}, \mathrm{R} C\rangle \geq 1$ for all blocks $\mathfrak{b} \in \mathcal{B} \ell(\mathfrak{g})$ contained in $C$.

Proof. Let $D^{\omega}$ denote the vector with components $D_{p}^{\omega}=\omega(p)^{-1} \mathrm{~d}_{p}$ for $p \in \mathfrak{b}$. According to Eq. (2.5), one has for $\alpha \in \mathfrak{g}$ and $\mathfrak{b} \subseteq \mathrm{C}$

$$
\begin{aligned}
\sum_{q \in \mathfrak{b}} \mathrm{N}_{\mathfrak{b}}(\alpha)_{p}^{q} D_{q}^{\omega}= & \sum_{q} N_{\alpha p}^{q} \omega(q)^{-1} \mathrm{~d}_{q}=\omega(\alpha)^{-1} \omega(p)^{-1} \frac{S_{\alpha p}}{S_{00}} \\
& =\omega(\alpha)^{-1} \frac{S_{\alpha p}}{S_{0 p}} \omega(p)^{-1} \frac{S_{0 p}}{S_{00}}=\boldsymbol{\rho}_{\mathrm{RC}}(\alpha) D_{p}^{\omega}
\end{aligned}
$$

in case $p \in \mathfrak{b}$. In other words, $D^{\omega}$ is a common eigenvector of the matrices $\mathrm{N}_{\mathfrak{b}}(\alpha)$, with eigenvalue $\rho_{\mathrm{RC}}(\alpha)$. Since $D_{p}^{\omega} \neq 0$, one gets that $\langle\mathfrak{b}, \mathrm{RC}\rangle$, the multiplicity of $\boldsymbol{\rho}_{\mathrm{RC}}$ in $\Delta_{\mathfrak{b}}$, is at least 1 .

Corollary 17. The number of blocks contained in the Ramond class equals the number of blocks contained in the trivial class.

Proof. Since $\langle\mathfrak{b}, R\rangle \leq 1$ by Proposition 2 (since $R$ is central), while $\langle\mathfrak{b}, R\rangle \geq 1$ for $\mathfrak{b} \subseteq \mathfrak{g}^{\perp}$ by the above Lemma, we get $\langle\mathfrak{b}, R\rangle=1$ and

$$
\left|\mathcal{B} \ell_{\mathrm{R}}(\mathfrak{g})\right|=\sum_{\mathfrak{b} \subseteq \mathfrak{g}^{\perp}}\langle\mathfrak{b}, \mathbf{R}\rangle=\left|\mathcal{B} \ell_{\mathfrak{g}^{\perp}}(\mathfrak{g})\right|
$$

by Eq. (7.1), proving the claim. 
Roughly speaking, the above Corollary says that, in case the Ramond class is nontrivial, there is an equal number of bosonic and fermionic degrees of freedom in the corresponding deconstructed model.

Theorem 7. Every local FC set belongs to $\mathscr{L}_{i n t}^{+}$, i.e. $d_{\alpha} \in \mathbb{Z}$ for all $\alpha \in \mathfrak{g}$.

Proof. We shall prove that $\ell \mathfrak{g} \subseteq \mathfrak{g}^{\perp}$ for all $\ell \in(\mathbb{Z} / N \mathbb{Z})^{\times}$. Since $\ell \mathfrak{g} \subseteq \ell \mathfrak{g}^{\perp}$ as a consequence of locality, and two classes are either equal or disjoint, this will imply the assertion by Lemma 21.

In case $\mathfrak{g}$ is a twister, Eq.(6.3) gives $h_{\ell \alpha}-h_{\ell 0} \in \mathbb{Z}$ since $h_{\alpha} \in \mathbb{Z}$ for all $\alpha \in \mathfrak{g}$. But this is tantamount to $\ell \mathfrak{g} \subseteq \mathrm{R}$ by Lemma 30 , and since the Ramond class is trivial for a twister, we get that $\ell \mathfrak{g} \subseteq \mathfrak{g}^{\perp}$.

If $\mathfrak{g}$ is not a twister, then $\mathfrak{g}_{+}=\{\alpha \in \mathfrak{g} \mid \omega(\alpha)=1\}$ is a twister by Corollary 15, and the above argument shows that $\ell \mathfrak{g}_{+} \subseteq \mathfrak{g}_{+}^{\perp}$. Since $\mathfrak{g}_{+}^{\perp}=\mathfrak{g}^{\perp} \cup \mathrm{R}$ by Proposition 6 , this means that either $\ell \mathfrak{g}_{+} \subseteq \mathfrak{g}^{\perp}$ or $\ell \mathfrak{g}_{+} \subseteq \mathrm{R}$. But $\mathfrak{g}_{+} \subseteq \mathfrak{g}$ implies that $\ell \mathfrak{g}_{+}$is contained in the $\mathfrak{g}$-block $\ell \mathfrak{g}$, and because every block of a local FC set is contained in precisely one class, one has either $\ell \mathfrak{g} \subseteq \mathfrak{g}^{\perp}$ or $\ell \mathfrak{g} \subseteq \mathrm{R}$. Since $\mathfrak{g}$ is not a twister, there exists some $\alpha \in \mathfrak{g}$ of half-integral conformal weight, which implies that the conductor $N$ is even, and $h_{\ell \alpha}-h_{\ell 0}$ belongs to $\mathbb{Z}+\frac{1}{2}$ according to Eq. (6.3): this contradicts $\ell \mathfrak{g} \subseteq \mathrm{R}$ as a consequence of Lemma 30, and thus proves that $\ell \mathfrak{g} \subseteq \mathfrak{g}^{\perp}$ in this case as well.

As surprising as this result may look at first sight, it is actually easy to understand from a vantage point of view. Indeed, it follows from Lemma 27 that the elements of a local FC set $\mathfrak{g} \in \mathscr{L}_{\text {loc }}$ are the simple objects of a symmetric monoidal subcategory of the modular tensor category associated to the conformal model. By a result of Deligne [11], such categories can be identified with the representation category of some (finite) group $G$, hence the algebra $\mathcal{V}_{\mathfrak{g}}$ associated to $\mathfrak{g}$ is isomorphic to the character ring of the group $G$, and in particular the (quantum) dimension of its elements are rational integers. But this is not the end of the story, for all general properties of character rings should apply to $\mathcal{V}_{\mathfrak{g}}$ in this case, so the following statements [24,26] should hold for $\mathfrak{g} \in \mathscr{L}_{\text {loc }}$ and $\alpha \in \mathfrak{g}:$

(1) the extent of any $\mathfrak{g}$-class is a rational integer dividing $\llbracket \mathfrak{g}^{\perp} \rrbracket$;

(2) $\alpha(\mathrm{C})=0$ for some class $\mathrm{C} \in \mathscr{C} \ell(\mathfrak{g})$ iff $\mathrm{d}_{\alpha}>1$;

(3) $|\mathrm{Z}(\mathfrak{g})|^{2} d_{\alpha}^{2}$ is an integer divisor of $\llbracket \mathfrak{g}^{\perp} \rrbracket^{2}$;

(4) if $d_{\alpha}^{2}$ is coprime to the ratio $\frac{\llbracket \mathfrak{g}^{\perp} \rrbracket}{\llbracket \mathrm{C} \rrbracket}$ for some class $\mathrm{C} \in \mathscr{C} \ell(\mathfrak{g})$, then either $|\alpha(\mathrm{C})|=\mathrm{d}_{\alpha}$ or $\alpha(\mathrm{C})=0$.

All these assertions are well-known properties of character rings, e.g. the first one just states that the size of a conjugacy class is an integer dividing the order of the group, while the third one is equivalent to Ito's famous theorem [24]. What is really amazing is that, as suggested by extensive computational evidence, they seem to hold for all members of $\mathscr{L}_{\text {int }}$, even in cases when there is no finite group with a suitable character ring. From this point of view, it seems fair to say that elements of $\mathscr{L}_{\text {int }}$ describe "character rings" of some natural generalization of the group concept. This interpretation seems the more reasonable as a host of group theoretical notions may be generalized to arbitrary elements of $\mathscr{L}_{\text {int }}$ : we have already encountered Abelian (Definition 9) and nilpotent FC sets (Definition 11), but the notion of (super)solvability can also be generalized to $\mathscr{L}_{\text {int. }}$ 
Definition 13. An FC set $\mathfrak{g} \in \mathscr{L}_{\text {int }}$ is solvable (supersolvable) if there exists a chain

$$
\{0\}=\mathfrak{g}_{0} \subseteq \mathfrak{g}_{1} \subseteq \cdots \subseteq \mathfrak{g}_{n}=\mathfrak{g}
$$

of FC sets $\mathfrak{g}_{i} \in \mathscr{L}$ such that $\llbracket \mathfrak{g}_{i} \rrbracket$ equals $\llbracket \mathfrak{g}_{i-1} \rrbracket$ times a prime power (a prime number) for $i=1, \ldots, n$.

For local FC sets this is clearly equivalent to the (super)solvability of the corresponding group, and one may speculate whether some kind of analogue of the Feit-Thompson theorem holds, i.e. whether $\mathfrak{g} \in \mathscr{L}_{\text {int }}$ is solvable provided $\llbracket \mathfrak{g} \rrbracket$ is odd.

\section{FC sets and Orbifold Deconstruction}

As mentioned in the introduction, the basic observation underlying orbifold deconstruction is that each orbifold model has a distinguished set of primaries, the 'vacuum block', which consists of those primaries that originate from the vacuum, and which has very special properties: it is closed under the fusion product, and all of its elements have integral conformal weight and quantum dimension. Such sets of primaries have been dubbed 'twisters' in [6], where it has been argued that each such twister gives rise to a different deconstruction, realizing the given model as a different orbifold, with possibly different twist group and/or original model. In particular, to each twister is associated a (finite) group, the twist group of the corresponding orbifold realization, which could be determined from the knowledge of the twister $[3,6]$.

But this is far from the end of the story, because each twister determines so-called 'twist classes' and 'blocks'. Twist classes are sets of primaries arising from the twisted modules contained in the same twisted sector, hence such classes are in one-to-one correspondence with conjugacy classes of the twist group. On the other hand, blocks correspond to orbits of the twist group on the set of all twisted modules, and since the action of the twist group does not change the twisted sector to which a given twisted module belongs to, each block should be contained in a unique twist class, and in particular, the vacuum block is contained in the trivial class (the 'untwisted sector').

All of this fits together nicely with the results of the present work. Because of its inclusion into the trivial class, the vacuum block is a local FC set all of whose elements have integral conformal weight (the integrality of quantum dimensions being, according to Theorem 7, an automatic consequence of locality), hence a twister as defined in Sect. 7. Twist classes of a twister $\mathfrak{g}$ are just the $\mathfrak{g}$-classes, while its blocks are the classes of its dual $\mathfrak{g}^{\perp}$, and that each block is contained in a unique class follows from Lemma 25 .

Of the several numerical attributes characterizing twist classes and blocks, a most important is the extent of a class as defined by Eq. (3.4). It follows from the deconstruction procedure that the extent has a natural interpretation in terms of the order of the centralizer of (an arbitrary element of) the conjugacy class corresponding to the twist class. In particular, the extent of the trivial class equals the order of the corresponding twist group, and the extent of any class should be a rational integer dividing it. And indeed, this holds not only for twisters, but (at least conjecturally, see the discussion after Theorem 7) for the much larger class of integral FC sets.

Since they originate from twisted modules related by the action of the twist group, the primaries contained in any given block of an orbifold are strongly interconnected. In particular, the ratios of their (quantum) dimensions should be rational numbers, while their conformal weights can only differ by integer multiples of $\frac{1}{n}$, where $n$ is the order of (any element of) the conjugacy class corresponding to the twist class containing the 
given block. That the first of these assertions holds follows from Lemma 23 (and is valid in the much broader context of integral FC sets), while a special case of the second one (valid for central classes) is the content of Lemma 30.

Any given conformal model may have several different twisters, and these can be ordered by inclusion. While the intersection of two twisters is clearly a twister again, i.e. twisters form a (finite) meet-semilattice, there can be several maximal twisters that are not contained properly in any other, hence (in general) twisters do not form a lattice. But if one fixes a given twister $\mathfrak{g}$ and considers only those twisters $\mathfrak{h}$ that are contained in it, these will form a lattice $\mathscr{L}_{\mathfrak{g}}=\{\mathfrak{h} \in \mathscr{L} \mid \mathfrak{h} \subseteq \mathfrak{g}\}$. Because the deconstruction corresponding to a twister $\mathfrak{h} \in \mathscr{L}_{\mathfrak{g}}$ is a partial deconstruction of that corresponding to $\mathfrak{g}$, with a twist group which is a homomorphic image (i.e. factor group) of that of $\mathfrak{g}$, the lattice $\mathscr{L}_{\mathfrak{g}}$ should be isomorphic to the lattice of normal subgroups of some finite group, and because the latter is a modular lattice, the same should hold for $\mathscr{L}_{\mathfrak{g}}$. That this is indeed so follows from Theorem 2, since any sublattice of a modular lattice is itself modular. While the above argument shows that the lattice $\mathscr{L}_{\mathfrak{g}}$ should be isomorphic to the lattice of normal subgroups of some finite group in case $\mathfrak{g}$ is a twister, it is far from clear whether this generalizes to other types of FC sets, like integral or nilpotent ones: the answer is trivially yes for Abelian $\mathfrak{g}$, since in that case all classes are central, hence $\mathfrak{g}$ coincides with the character ring of its center $\mathrm{Z}(\mathfrak{g})$, but in general this seems to be a deep and interesting question.

Given a twister $\mathfrak{g}$, one could ask which group theoretic properties of the corresponding twist group can be inferred from the knowledge of $\mathfrak{g}$ ? Orbifold deconstruction tells us that attributes like the order, the dimension of the (complex) irreducible representations, the size of the conjugacy classes, etc., can be read off from properties of $\mathfrak{g}$, and in particular, one can decide simply whether the twist group is Abelian, nilpotent or soluble. The generalization of the relevant criteria to arbitrary FC sets are being given by Definitions 9, 11 and 13. In a similar vein, one may ask for the structure of the center of the twist group, which notion turns out to be captured by the center $Z(\mathfrak{g})$ of $\mathfrak{g}$. Note that all the above results and notions do make sense not only for twisters, but for arbitrary FC sets, providing thus an interesting extension of many classical concepts from group theory.

It should be stressed that, would any of the above mentioned properties fail, the group theoretic interpretation of the deconstruction procedure would be inherently flawed. That so many non-trivial statements follow from the very definition of twisters is a strong supporting evidence that the latter do indeed capture essential features of vacuum blocks of orbifold models. But one may go further, and speculate that the larger class of integral FC sets might be related to some kind of generalized orbifolds, obtained from some suitable generalization of the group concept (which should be a true generalization, for there are known examples of integral FC sets that are not character rings of any finite group). One of the motivations of the present work was, besides providing a formal foundation of the ideas presented in $[3,6]$, to unravel such possible generalizations.

\section{Summary and Outlook}

As we have seen, fusion closed sets of primaries of a conformal model (or modular tensor category) have a fairly deep structure, generalizing many aspects of the character theory of finite groups. Of course, this is no accident, since vacuum blocks of orbifold models, which correspond on general grounds to the character ring of the twist group, form a special class of FC sets. But it turns out that the parallel with character theory goes much further, even for FC sets that have no group theoretic origin. Many classical notions from 
group theory (like nilpotency, solubility, etc.) may be generalized to arbitrary FC sets, and the corresponding properties go over almost verbatim to this more general setting. In this respect, a major goal of the present work is to illustrate this close analogy with classical group theory, but it should be stressed that FC sets are more than just some fancy generalization of the group concept, since they possess genuinely new properties, as exemplified by Lemma 24 or the reciprocity relations Eq. (3.19).

Of course, the results presented fall short of giving due account of all important aspects of FC sets relevant to orbifold deconstruction. In particular, the $\lambda$-ring structure of local FC sets, which is of utmost importance for the identification of twist groups $[3,6]$, has not been discussed. While fundamental, we felt that the presentation of these issues could dim the overall pattern, and have consequently decided to relegate their discussion to some future work.

Finally, a few words about the mathematics involved. While many of the results presented might be formulated in the language of (unitary) modular tensor categories $[2,14,16,17,28,33]$, in our opinion this could obscure the analogies with group theory, which were among the major motivations of this work. Besides this, some of the more interesting results and conjectures, like Lemma 22 or Conjecture 3, seem difficult to formulate using category theory solely. For these reasons, we opted for a mode of exposition closer to that of classical texts $[24,32]$ on representation theory.

Funding Open access funding provided by Eötvös Loránd University.

Open Access This article is licensed under a Creative Commons Attribution 4.0 International License, which permits use, sharing, adaptation, distribution and reproduction in any medium or format, as long as you give appropriate credit to the original author(s) and the source, provide a link to the Creative Commons licence, and indicate if changes were made. The images or other third party material in this article are included in the article's Creative Commons licence, unless indicated otherwise in a credit line to the material. If material is not included in the article's Creative Commons licence and your intended use is not permitted by statutory regulation or exceeds the permitted use, you will need to obtain permission directly from the copyright holder. To view a copy of this licence, visit http://creativecommons.org/licenses/by/4.0/.

Publisher's Note Springer Nature remains neutral with regard to jurisdictional claims in published maps and institutional affiliations.

\section{References}

1. Alperin, J.L., Bell, R.B.: Groups and Representations. Graduate Text in Mathematics, vol. 162. Springer, New York (1995)

2. Bakalov, B., Kirillov, A.A.: Lectures on Tensor Categories and Modular Functors. University Lecture Series, vol. 21. AMS, Providence (2001)

3. Bantay, P.: Orbifold deconstruction: a computational approach. Contemp. Math. 753, 1-15 (2020)

4. Bantay, P.: Simple current extensions and mapping class group representations. Int. J. Mod. Phys. A 13, 199-208 (1998)

5. Bantay, P.: The kernel of the modular representation and the Galois action in RCFT. Commun. Math. Phys. 233, 423-438 (2003)

6. Bantay, P.: A short guide to orbifold deconstruction. SIGMA 15, 027-037 (2019)

7. Coste, A., Gannon, T.: Phys. Lett. B 323, 316 (1994)

8. Crawley, P., Dilworth, R.P.: Algebraic Theory of Lattices. Prentice-Hall, Englewood Cliffs (1973)

9. Day, A., Pickering, D.: The coordinatization of arguesian lattices. Trans. Amer. Math. Soc. 278, 507-522 (1983)

10. de Boere, J., and Goeree, J.: Commun. Math. Phys. 139, 267 (1991)

11. Deligne, P.: Categories tannakiennes. In The Grothendieck Festschrift, Progr. Math. 87, pp. 111-195. Birkhauser, (1990)

12. Dixon, L.J., Friedan, D., Martinec, E.J., Shenker, S.H.: The conformal field theory of orbifolds. Nucl. Phys. B 282, 13-73 (1987) 
13. Di Francesco, P., Mathieu, P., Sénéchal, D.: Conformal Field Theory. Springer, New York (1997)

14. Drinfeld, V., Gelaki, S., Nikshych, D., Ostrik, V.: On braided fusion categories I. Sel. Math. 16(1), 1-119 (2010)

15. Frenkel, I., Lepowsky, J., Meurman, A.: Vertex Operator Algebras and the Monster. Pure and Applied Mathematics, vol. 134. Academic Press, New York (1988)

16. Fröhlich, J., Fuchs, J., Runkel, I., Schweigert, C.: Defect lines, dualities, and generalised orbifolds. In XVI International Congress on Mathematical Physics, Prague, August 3-8, 2009, (2009)

17. Fröhlich, J., Fuchs, J., Runkel, I., Schweigert, Ch.: Correspondences of ribbon categories. Adv. Math. 199, 192-329 (2006)

18. Fuchs, J., Schellekens, A.N., Schweigert, C.: A matrix S for all simple current extensions. Nucl. Phys. B 473, 323-366 (1996)

19. Fulton, W., Harris, J.: Representation Theory: A First Course. GTM, vol. 129. Springer, Berlin (1991)

20. Gantmacher, F.: The Theory of Matrices. AMS Chelsea Publishing, New York (1959)

21. Ginsparg, P.: Curiosities at $c=1$. Nucl. Phys. B 295, 153 (1988)

22. Grätzer, G.: Lattice Theory: Foundation. Birkhäuser Verlag, Basel (2011)

23. Intriligator, K.A.: Nucl. Phys. B 332, 541 (1990)

24. Isaacs, I.M.: Character Theory of Finite Groups. Pure and Applied Mathematics, vol. 22. Academic Press, New York (1977)

25. Jonsson, B.: On the representation of lattices. Math. Scand. 1, 193-206 (1953)

26. Lux, K., Pahlings, H.: Representations of Groups A Computational Approach, volume 124 of Cambridge Studies in Advanced Mathematics. Cambridge University Press, (2010)

27. Moore, G., Seiberg, N.: Lectures on RCFT, volume Physics, Geometry and Topology. of NATO ASI Series (Series B: Physics), vol 238, pp. 263-361. Springer, Boston (1990)

28. Müger, M.: On the structure of braided crossed $G$-categories. in V. G. Turaev (editor), Homotopy Quantum F. Theory, Appendix 5, pp. 221-235. European Mathematical Society (2010)

29. Pálfy, P .P., Szabó, Cs: On the representation of lattices. Algebra Univ. 33, 191-195 (1995)

30. Schellekens, A.N., Yankielowicz, S.: Phys. Lett. B 227, 387 (1989)

31. Schellekens, A.N., Yankielowicz, S.: Int. J. Mod. Phys. A 5, 2903-2952 (1990)

32. Serre, J.P.: Linear Representations of Finite Groups. Graduate Text in Mathematics, vol. 42. Springer, Berlin (1977)

33. Turaev, V.G.: Quantum Invariants of Knots and 3-Manifolds. Studies in Mathematics, vol. 18. de Gruyter, Berlin (1994)

34. Verlinde, E.: Fusion rules and modular transformations in 2D conformal field theory. Nucl. Phys. B 300 , 360-376 (1988)

35. von Neumann, J.: Algebraic theory of continuous geometries. Proc. Nat. Acad. Sci. U.S.A. 23, 16-22 (1937)

Communicated by C. Schweigert 